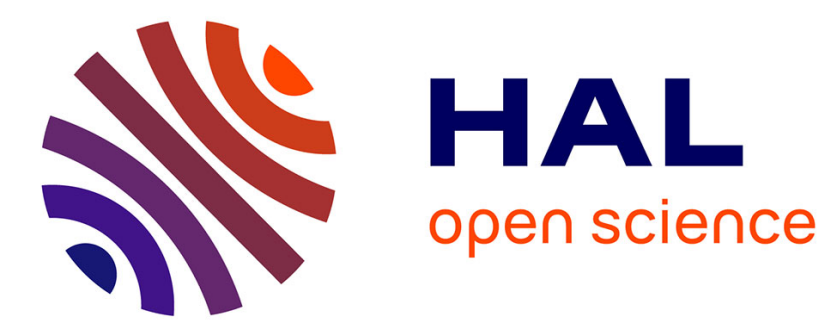

\title{
Symbolic analysis of terrorist fraud resistance
}

Alexandre Debant, Stéphanie Delaune, Cyrille Wiedling

\section{To cite this version:}

Alexandre Debant, Stéphanie Delaune, Cyrille Wiedling. Symbolic analysis of terrorist fraud resistance. Computer Security - ESORICS 2019 - 24th European Symposium on Research in Computer Security, Sep 2019, Luxembourg, Luxembourg. hal-02171218

\section{HAL Id: hal-02171218 \\ https://hal.inria.fr/hal-02171218}

Submitted on 10 Jul 2019

HAL is a multi-disciplinary open access archive for the deposit and dissemination of scientific research documents, whether they are published or not. The documents may come from teaching and research institutions in France or abroad, or from public or private research centers.
L'archive ouverte pluridisciplinaire HAL, est destinée au dépôt et à la diffusion de documents scientifiques de niveau recherche, publiés ou non, émanant des établissements d'enseignement et de recherche français ou étrangers, des laboratoires publics ou privés. 


\title{
Symbolic analysis of terrorist fraud resistance *
}

\author{
Alexandre Debant ${ }^{1}$, Stéphanie Delaune ${ }^{1}$, and Cyrille Wiedling ${ }^{2}$ \\ 1 Univ Rennes, CNRS, IRISA, France \\ \{alexandre.debant, stephanie.delaune\}@irisa.fr \\ 2 DGA MI, Bruz, France \\ cwiedling@gmail.com
}

\begin{abstract}
Distance-bounding protocols aim at preventing several kinds of attacks, amongst which terrorist fraud, where a far away malicious prover colludes with an attacker to authenticate once, without giving him any advantage for future authentication. In this paper, we consider a symbolic setting and propose a formal definition of terrorist fraud, as well as two reduction results. When looking for an attack, we can first restrict ourselves to consider a particular (and quite simple) topology. Moreover, under some mild hypotheses, the far away malicious prover has a best strategy on which we can focus on when looking for an attack. These two reduction results make possible the analysis of terrorist fraud resistance using an existing verification tool. As an application, we analyse several distance-bounding protocols, as well as some contactless payment protocols using the ProVerif tool.
\end{abstract}

\section{Introduction}

Contactless devices deployed today in ticketing and building access-control applications are supposed to make our life easier but they also make possible new kinds of attacks, e.g. relay attacks. An attacker can use two transponders (two mobile phones could be sufficient) in order to relay over a large distance the information between e.g. a card and an access card reader. As a result, an unauthorised person will be able to enter a building using an access card located far away and possibly still in the pocket of his holder. With the deployment of contactless systems, ensuring "proximity authentication", through the use of secure protocols, is an important goal.

Relay attacks cannot be prevented by traditional cryptographic protocols. One possible defence is distance bounding protocols. The main goal of a distance bounding protocol consists of ensuring that a prover is within a close distance to a verifier by timing the round-trip delay of a cryptographic challenge-response exchange. Therefore the security of these protocols is based on the physical limits of communication: transmission can not go faster than the speed of light. Since they have been introduced by Brands and Chaum in 1993 [8], many protocols

\footnotetext{
^ This work has been partially supported by the European Research Council (ERC) under the European Union's Horizon 2020 research and innovation program (grant agreement No 714955-POPSTAR).
} 
have been designed and analysed against various threats. In general, distance bounding protocols shall resist to distance fraud: a malicious prover should not be able to successfully complete a session with an honest verifier who is far away (even with the help of some honest provers in the neighbourhood - so called distance hijacking). They should also resist to mafia fraud where typically an attacker abuses a far away prover to pass the protocol. In most cases, this is achieved by relaying messages between the prover and the verifier (the so-called relay attack). A more subtle notion is the notion of terrorist fraud. Here, a far away malicious prover colludes with an attacker who is close to the verifier to pass the protocol on his behalf. Such a scenario may occur if a legitimate worker want to enable a third party to access his office, located in a restricted area, when he is away. To prevent such behaviours, the protocol is said resistant to a terrorist fraud if this help is actually reusable meaning that the third party can use this extra information to impersonate the prover later on. The rationale is that a malicious prover will not accept to give such an advantage to his accomplice, and thus will not accept to collude with the attacker. This type of attack is very tricky and rather difficult to model and analyse since it requires to consider "terrorist" provers that are not fully dishonest in the sense that they are not willing for instance to reveal their credentials.

Formal symbolic modelling and analysing techniques have proved their usefulness for verifying security protocols, and nowadays several verification tools exist, e.g. ProVerif [5,6], Tamarin [26]. Since the seminal paper by Dolev-Yao in [16], a lot of progress has been done in the area of formal symbolic verification and it is now a common good practice to formally analyse a protocol using these techniques before their deployment. In this so-called Dolev-Yao model, messages are transmitted without introducing any delay preventing us to use this model to analyse protocols for which transmission delay plays an important role. To overcome this limitation, getting some inspiration from earlier works (e.g. [25, $4,28]$ ), some recent works have proposed to incorporate new features in existing symbolic models $[23,14,11,13]$, making the analysis of distance bounding protocols possible relying on existing verification tools (e.g. ProVerif, Tamarin).

Our contributions. In this paper, distance bounding protocols are modelled using the calculus we introduced in [14]. This calculus shares some similarities with the applied pi calculus $[1,6]$, a well-established process algebra for modelling cryptographic protocols. Within this framework, we propose a formal definition of terrorist fraud. We will see that this notion is tricky and complex and require a quantification over all the topologies, but also another one to consider all the possible terrorist provers. Due to this, such a security property can not be analysed using techniques deployed in e.g. [23,14,13]. Our main contribution is to provide reduction results to reduce the number of topologies we have to consider during our analysis, and more importantly to reduce the possible behaviours of our terrorist prover. We will see that under some reasonable conditions, we are able to reduce the number of topologies to be considered to one (involving at most 4 participants), and the best strategy for the terrorist prover can also be fixed without missing any attack. Then, an interesting consequence of our 
results is that, following the approach used e.g. in $[14,11]$, it becomes possible to rely on the automatic verification tool ProVerif (originally developed to analyse traditional security protocols) to analyse terrorist fraud in various distance bounding protocols. All the omitted proofs are available in the full version [15].

Related works. Several attempts have been made in the computational model to formalise terrorist fraud, e.g. [17, 2, 18, 30]. Avoine et al. [2] introduce a unified framework for clarifying the situation and make possible comparison between protocols. Since then, several formal definitions of terrorist fraud have been proposed $[18,30]$, as well as protocols supposed to achieve this level of security, e.g. $[22,9,3]$. In contrast, the only definition we are aware of in the symbolic model is the one proposed by Chothia et al in [11]. However, such a definition falls short when modelling behaviours of terrorist provers (see Section 3). Independently of our work, Mauw et al proposed a definition more in line with the one we considered here [24]. However, their work falls short when it comes to the automation of security analysis (see Section 4.3).

\section{Model for distance bounding protocols}

In this section, we introduce the process calculus we rely on to describe distance bounding protocols [14]. It shares some similarities with the applied pi calculus used e.g. by the ProVerif verification tool [6].

\section{$2.1 \quad$ Messages}

As usual in the symbolic setting, we model messages through a term algebra. We consider both equational theories and reduction relations to represent the properties of the cryptographic primitives.

Term algebra. We consider two infinite and disjoint sets of names: $\mathcal{N}$ is the set of basic names, which are used to represent keys, nonces, whereas $\mathcal{A}$ is the set of agent names, i.e. names which represent the agents identities. We consider an infinite set $\Sigma_{0}$ of constant symbols that are used to represent values known by the attacker, as well as two infinite and disjoint sets of variables, denoted $\mathcal{X}$ and $\mathcal{W}$. Variables in $\mathcal{X}$ refer to unknown parts of messages expected by participants while variables in $\mathcal{W}$ are used to store messages learnt by the attacker.

We assume a signature $\Sigma$, i.e. a set of function symbols together with their arity. The elements of $\Sigma$ are split into constructor and destructor symbols, i.e. $\Sigma=\Sigma_{c} \uplus \Sigma_{d}$. We denote $\Sigma^{+}=\Sigma \cup \Sigma_{0}$, and $\Sigma_{c}^{+}=\Sigma_{c} \cup \Sigma_{0}$. Given a signature $\mathcal{F}$, and a set of atomic data $\mathrm{A}$, we denote by $\mathcal{T}(\mathcal{F}, \mathrm{A})$ the set of terms built from atomic data $\mathrm{A}$ by applying function symbols in $\mathcal{F}$. A constructor term is a term in $\mathcal{T}\left(\Sigma_{c}^{+}, \mathcal{N} \cup \mathcal{A} \cup \mathcal{X}\right)$. We denote $\operatorname{vars}(u)$ the set of variables that occur in a term $u$. A message is a constructor term $u$ that is ground, i.e. such that vars $(u)=\emptyset$. The application of a substitution $\sigma$ to a term $u$ is written $u \sigma$. We denote $\operatorname{dom}(\sigma)$ its domain, and $i m g(\sigma)$ its image. The positions of a term are defined as usual. 
Example 1. We consider the signature $\Sigma_{\text {ex }}=\{\mathrm{kdf} / 3$, shk $/ 2$, ok $/ 0$, eq $/ 2$, ans $/ 3\}$. The symbol kdf models a key derivation function, shk is used to model a key shared between 2 agents. The symbols ok and eq are used to model equality tests, and ans is a function symbol that is used to model the answer provided by the prover. Another signature useful to model the exclusive-or operator is $\Sigma_{\text {xor }}=\{\oplus, 0\}$. Among all the symbols in $\Sigma_{\text {ex }} \cup \Sigma_{\text {xor }}$ only eq is a destructor.

Equational theory. Following the approach developed in [6], constructor terms are subject to an equational theory allowing us to model the algebraic properties of the primitives. It consists of a finite set of equations of the form $u=v$ where $u, v \in \mathcal{T}\left(\Sigma_{c}, \mathcal{X}\right)$, and induces an equivalence relation $=_{\mathrm{E}}$ over constructor terms. ${ }^{1}$

Example 2. To reflect the algebraic properties of the exclusive-or operator, we may consider the equational theory $\mathrm{E}_{\mathrm{xor}}$ generated by the following equations:

$$
(x \oplus y) \oplus z=x \oplus(y \oplus z) \quad x \oplus y=y \oplus x \quad x \oplus 0=x \quad x \oplus x=0 .
$$

Rewriting rules. As in [6], we also give a meaning to destructor symbols. This is done through a set of rewrite rules of the form $\mathrm{g}\left(t_{1}, \ldots, t_{n}\right) \rightarrow t$ where $\mathrm{g} \in \Sigma_{d}$, and $t, t_{1}, \ldots, t_{n} \in \mathcal{T}\left(\Sigma_{c}, \mathcal{X}\right)$. A term $u$ can be rewritten in $v$ if there is a position $p$ in $u$, and a rewrite rule $\mathrm{g}\left(t_{1}, \ldots, t_{n}\right) \rightarrow t$ such that $\left.u\right|_{p}=_{\mathrm{E}} \mathrm{g}\left(t_{1}, \ldots, t_{n}\right) \theta$ for some substitution $\theta$, and $v=u[t \theta]_{p}$ i.e. $u$ in which the term at position $p$ has been replaced by $t \theta$. Moreover, we assume that $t_{1} \theta, \ldots, t_{n} \theta$ as well as $t \theta$ are constructor terms. We only consider sets of rewrite rules that yield a convergent rewriting system (modulo E), and we denote $u \downarrow$ the normal form of a term $u$.

For modelling purposes, we split the signature $\Sigma$ into two parts, $\Sigma_{\text {pub }}$ and $\Sigma_{\text {priv }}$, and we denote $\Sigma_{\text {pub }}^{+}=\Sigma_{\text {pub }} \cup \Sigma_{0}$. An attacker builds messages by applying public symbols to terms he knows and that are available through variables in $\mathcal{W}$. Formally, a computation done by the attacker is a recipe, i.e. a term in $\mathcal{T}\left(\Sigma_{\text {pub }}^{+}, \mathcal{W}\right)$.

Example 3. Among symbols in $\Sigma_{\text {ex }} \cup \Sigma_{\text {xor }}$, only shk is in $\Sigma_{\text {priv }}$. The property of the symbol eq is reflected by the rule eq $(x, x) \rightarrow$ ok. Note that eq $(u, v)$ reduces to a message if, and only if, $u={ }_{\mathrm{E}} v$. A typical signature used to model security protocols is $\Sigma_{\text {enc }}=\{$ senc, sdec $\}$. Depending on whether we want to model a decryption algorithm that may fail or not, we can either consider sdec as a destructor together with the rewrite rule $\operatorname{sdec}(\operatorname{senc}(x, y), y) \rightarrow x$, or consider both symbols as constructors, together with equation $\operatorname{sdec}(\operatorname{senc}(x, y), y)=x$. In the latter case, $\operatorname{sdec}(c, k)$ will be considered as a "valid" message.

Given a set $\mathcal{U}$ of equations between terms, $\sigma$ is a unifier for $\mathcal{U}$ if $u_{1} \sigma \downarrow={ }_{\mathrm{E}} u_{2} \sigma \downarrow$ and both $u_{1} \sigma \downarrow$ and $u_{2} \sigma \downarrow$ are constructor terms for any $u_{1}=u_{2} \in \mathcal{U}$. We denote by $\operatorname{csu}(\mathcal{U})$ a set of unifiers for $\mathcal{U}$ which is also complete, i.e. such that for any $\sigma$ unifier of $\mathcal{U}$, there exists $\theta \in \operatorname{csu}(\mathcal{U})$ such that $\sigma=_{\mathrm{E}} \tau \circ \theta$ for some $\tau$.

Example 4. Let $\mathcal{U}=\left\{x_{0}=m_{0}, x_{1}=k \oplus x_{0} ; x_{\text {ok }}=\right.$ eq $\left(x_{\text {rep }}\right.$, ans $\left.\left.\left(c, x_{0}, x_{1}\right)\right)\right\}$ with $k=\operatorname{shk}\left(p_{0}, v_{0}\right)$, and $m_{0}=\operatorname{kdf}\left(k, n_{V}, x_{N}\right)$. We have that $\operatorname{csu}(\mathcal{U})=\{\theta\}$ where $\theta$ is the substitution: $x_{0} \mapsto m_{0} ; x_{1} \mapsto k \oplus m_{0} ; x_{\text {rep }} \mapsto \operatorname{ans}\left(c, m_{0}, k \oplus m_{0}\right) ; x_{\text {ok }} \mapsto$ ok.

\footnotetext{
${ }^{1}$ We only consider non-trivial theories, i.e. there exist $u$ and $v$ such that $u \neq \neq_{\mathrm{E}} v$.
} 


\subsection{Protocols}

Protocols are modelled through processes that may receive and send messages.

Syntax. We consider the following grammar:

$P, Q=0|\operatorname{in}(x) . P|$ in $^{<t}(x) . P \mid$ let $x=v$ in $P \mid$ new $n . P|\operatorname{out}(u) . P|$ reset. $P$

where $x \in \mathcal{X}, n \in \mathcal{N}, u \in \mathcal{T}\left(\Sigma_{c}^{+}, \mathcal{X} \uplus \mathcal{N} \uplus \mathcal{A}\right), v \in \mathcal{T}\left(\Sigma^{+}, \mathcal{X} \uplus \mathcal{N} \uplus \mathcal{A}\right)$ and $t \in \mathbb{R}_{+}$.

We write $f v(P)$ (resp. $f n(P))$ for the set of free variables (resp. names) occurring in $P$, i.e. the set of variables (resp. names) that are not in the scope of an input or a let (resp. a new). In this work, we only consider 2-party protocols, and thus we consider parameterised processes, denoted $P\left(z_{0}, z_{1}\right)$, where $z_{0}$ and $z_{1}$ are variables from a special set $\mathcal{Z}$ (disjoint from $\mathcal{X}$ and $\mathcal{W}$ ). Intuitively, $z_{0}$ and $z_{1}$ will be instantiated by agent names: $z_{0}$ corresponds to the name of the agent that executes the process, and $z_{1}$ will be his interlocutor. A role $R=P\left(z_{0}, z_{1}\right)$ is a parameterised process such that $f n(R)=\emptyset$ and $f v(R) \subseteq\left\{z_{0}, z_{1}\right\}$. A protocol is given by two roles, denoted $\mathrm{V}\left(z_{0}, z_{1}\right)$ and $\mathrm{P}\left(z_{0}, z_{1}\right)$, and named respectively the verifier role and the prover role. Moreover, we will assume that the verifier role ends with a special construct end $\left(z_{0}, z_{1}\right)$ allowing us to see when he has completed his role and with whom. Formally, it simply means that, in the verifier role $\mathrm{V}\left(z_{0}, z_{1}\right)$, the process 0 has been replaced by end $\left(z_{0}, z_{1}\right)$.

Example 5. As a running example and for illustrative purposes, we consider a strengthened version of the Hancke and Kuhn distance bounding protocol [20] (as briefly described in [29]). It relies on the use of a keyed public pseudo-random function (modelled as a free function symbol here) and the exclusive-or operator.

$$
\begin{aligned}
& \text { 1. } V \rightarrow P: N_{V} \text { 3. } V \rightarrow P: c_{i} \\
& \text { 2. } P \rightarrow V: N_{P} \\
& \text { 4. } P \rightarrow V: \quad\left\{\begin{array}{l}
i^{\text {th }} \text { bit of } \operatorname{kdf}\left(k, N_{V}, N_{P}\right) \text { if } c_{i}=0 \\
i^{\text {th }} \text { bit of } k \oplus \operatorname{kdf}\left(k, N_{V}, N_{P}\right) \text { if } c_{i}=1
\end{array}\right.
\end{aligned}
$$

The protocol starts with both parties transmitting to each other their own nonce. Then, the verifier initiates the rapid phase during which the time measurement is performed. The verifier generates and sends a random bit $c_{i}$, and the prover has to reply immediately with the $i^{\text {th }}$ bit of $\operatorname{kdf}\left(k, N_{V}, N_{P}\right)$ if $c_{i}=0$ and the $i^{\text {th }}$ bit of $k \oplus \operatorname{kdf}\left(k, N_{V}, N_{P}\right)$ otherwise. This rapid exchange is repeated a fixed number of times, and if enough correct answers are received within a sufficiently short time after the corresponding challenge $c_{i}$ has been sent out, then the verifier is convinced that the prover is located in its vicinity. In our setting, this gives us:

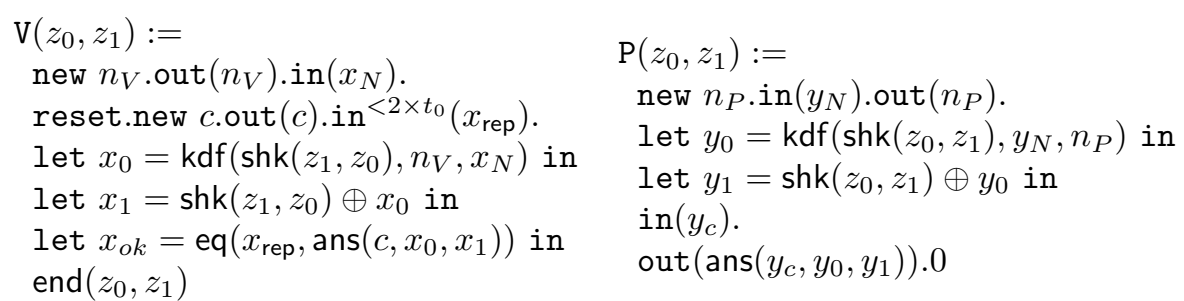


Symbolic analysis does not allow one to reason at the bit level, and thus, as done in e.g. $[23,14,11,13]$, all the challenge bits $c_{i}$ are collapsed into a single challenge/response exchange using a nonce. Furthermore, operations performed at the bit level are abstracted too. The response is therefore abstracted by an uninterpreted symbol of a function ans depending on both the challenge $c$ and the two precomputed values $y_{0}$ and $y_{1}$.

Topology. The semantics of our processes depends on their location. This is formally defined through the notion of topology.

Definition 1. A topology is a tuple $\mathcal{T}_{0}=\left(\mathcal{A}_{0}, \mathcal{M}_{0}\right.$, Loc $\left.{ }_{0}, v_{0}, p_{0}\right)$ where:

- $\mathcal{A}_{0} \subseteq \mathcal{A}$ is the finite set of agents composing the system;

- $\mathcal{M}_{0} \subseteq \mathcal{A}_{0}$ is the subset of agents that are malicious;

- Loc $_{0}: \mathcal{A}_{0} \rightarrow \mathbb{R}^{3}$ is a mapping defining the position of each agent in space.

- $p_{0}$ and $v_{0}$ are two agents in $\mathcal{A}_{0}$ that represent respectively the prover and the verifier w.r.t. which the analyse is performed.

In our model, the distance between two agents is expressed by the time it takes for a message to travel from one to another. Therefore, we consider

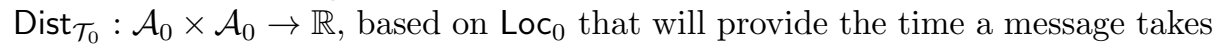
to travel between two agents. It is defined as follows:

$$
\operatorname{Dist}_{\mathcal{T}_{0}}(a, b)=\frac{\left\|\operatorname{Loc}_{0}(a)-\operatorname{Loc}_{0}(b)\right\|}{c_{0}} \text { for any } a, b \in \mathcal{A}_{0}
$$

with $\|\cdot\|: \mathbb{R}^{3} \rightarrow \mathbb{R}$ the Euclidean norm and $c_{0}$ the transmission speed. We suppose, from now on, that $c_{0}$ is a constant for all agents, and thus an agent $a$ can recover, at time $t$, any message emitted by any other agent $b$ before $t-\operatorname{Dist}_{\mathcal{T}_{0}}(a, b)$.

Example 6. When analysing a distance bounding protocol, we have to consider a class of topologies. Typically, a mafia fraud is an attack in which at least three agents are involved: an honest verifier, an honest prover, and an attacker. Of course, in general more agents may be involved, and the set $\mathcal{C}_{\mathrm{MF}}$ of all the mafia fraud topologies is simply defined as follows: any topology $\mathcal{T}=\left(\mathcal{A}_{0}, \mathcal{M}_{0}, \operatorname{Loc}_{0}, v_{0}, p_{0}\right)$ such that $v_{0}, p_{0} \in \mathcal{A}_{0} \backslash \mathcal{M}_{0}$.

Configuration. The semantics of our processes is given through a transition system defined over configurations. Given a topology $\mathcal{T}_{0}=\left(\mathcal{A}_{0}, \mathcal{M}_{0}, \operatorname{Loc}_{0}, v_{0}, p_{0}\right)$, a configuration $K$ over $\mathcal{T}_{0}$ is a tuple $(\mathcal{P} ; \Phi ; t)$, where:

- $\mathcal{P}$ is a multiset of extended process $\lfloor P\rfloor_{a}^{t_{a}}$ with $a \in \mathcal{A}_{0}$ and $t_{a} \in \mathbb{R}_{+}$;

$-\Phi=\left\{\mathrm{w}_{1} \stackrel{a_{1}, t_{1}}{\longrightarrow} u_{1}, \ldots, \mathrm{w}_{n} \stackrel{a_{n}, t_{n}}{\longrightarrow} u_{n}\right\}$ is an extended frame, i.e. a substitution such that $\mathrm{w}_{i} \in \mathcal{W}, u_{i} \in \mathcal{T}\left(\Sigma_{c}^{+}, \mathcal{N} \uplus \mathcal{A}\right), a_{i} \in \mathcal{A}_{0}$ and $t_{i} \in \mathbb{R}_{+}$for $1 \leq i \leq n$;

$-t \in \mathbb{R}_{+}$is the global time of the system.

A initial frame is a frame such that $t_{i}=0(1 \leq i \leq n)$, and an initial configuration is a configuration such that $t=0$. We write $\lfloor\Phi\rfloor{ }_{a}^{t}$ for the restriction of $\Phi$ to the agent $a$ at time $t$, i.e. :

$$
\lfloor\Phi\rfloor_{a}^{t}=\left\{\mathrm{w}_{i} \stackrel{a_{i}, t_{i}}{\longrightarrow} u_{i} \mid\left(\mathrm{w}_{i} \stackrel{a_{i}, t_{i}}{\longrightarrow} u_{i}\right) \in \Phi \text { and } a_{i}=a \text { and } t_{i} \leq t\right\} .
$$


Example 7. Continuing Example 5, we consider $\mathcal{T}_{0}=\left(\mathcal{A}_{0}, \mathcal{M}_{0}, \operatorname{Loc}_{0}, v_{0}, p_{0}\right)$ depicted below where $\mathcal{A}_{0}=\left\{p_{0}, v_{0}, p\right\}$, and $\mathcal{M}_{0}=\{p\}$.

The precise location of each agent is not relevant, only the distance between them matters. Here Dist $\mathcal{T}_{0}\left(p, v_{0}\right)<t_{0}$ whereas $\operatorname{Dist}_{\mathcal{T}_{0}}\left(p_{0}, v_{0}\right) \geq t_{0}$.

A possible initial configuration $K_{0}$ is given below:

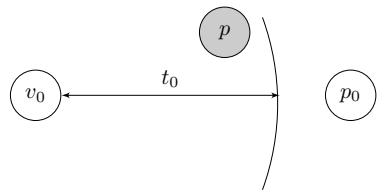

$$
\left(\left\lfloor\mathrm{P}\left(p_{0}, v_{0}\right)\right\rfloor_{p_{0}}^{0} \uplus\left\lfloor\mathrm{V}\left(v_{0}, p_{0}\right)\right\rfloor_{v_{0}}^{0} ;\left\{\mathrm{w}_{1} \stackrel{p, 0}{\longrightarrow} \operatorname{shk}\left(p, v_{0}\right), \mathrm{w}_{2} \stackrel{p, 0}{\longrightarrow} m_{0}, \mathrm{w}_{3} \stackrel{p, 0}{\longrightarrow} m_{1}\right\} ; 0\right)
$$

Here, $p_{0}$ and $v_{0}$ are honest agents playing respectively the prover's role and the verifier's role. The agent $p$ is a malicious prover whose shared key with $v_{0}$ is given to the attacker through $\mathrm{w}_{1}$. Here, we also assume that the attacker $p$ also knows $m_{0}=\operatorname{kdf}\left(\operatorname{shk}\left(p_{0}, v_{0}\right), n_{V}^{0}, n_{P}^{0}\right)$ and $m_{1}=\operatorname{shk}\left(p_{0}, v_{0}\right) \oplus \operatorname{kdf}\left(\operatorname{shk}\left(p_{0}, v_{0}\right), n_{V}^{0}, n_{P}^{0}\right)$. These messages coming from an older session may have been given to him by $p_{0}$ to let the attacker exceptionally authenticate on his behalf. A more realistic configuration will include other instances of these two roles and will probably give more knowledge to the attacker.

Semantics. We now recall the semantics of our calculus as defined in [14].

$$
\begin{aligned}
& \text { Tim } \quad(\mathcal{P} ; \Phi ; t) \longrightarrow \mathcal{T}_{0}(\operatorname{Shift}(\mathcal{P}, \delta) ; \Phi ; t+\delta) \quad \text { with } \delta \geq 0 \\
& \text { RST } \quad\left(\lfloor\text { reset.P }\rfloor_{a}^{t_{a}} \uplus \mathcal{P} ; \Phi ; t\right) \stackrel{a, \tau}{\longrightarrow} \mathcal{T}_{0}\left(\lfloor P\rfloor_{a}^{0} \uplus \mathcal{P} ; \Phi ; t\right) \\
& \text { OUT } \left.\quad\left(\lfloor\text { out }(u) . P\rfloor_{a}^{t_{a}}\right) \uplus \mathcal{P} ; \Phi ; t\right) \stackrel{a, \text { out }(u)}{\longrightarrow} \mathcal{T}_{0}\left(\lfloor P\rfloor_{a}^{t_{a}} \uplus \mathcal{P} ; \Phi \uplus\{\mathrm{w} \stackrel{a, t}{\longrightarrow} u\} ; t\right) \\
& \text { with } w \in \mathcal{W} \text { fresh } \\
& \text { LET } \quad\left(\lfloor\text { let } x=u \text { in } P\rfloor_{a}^{t_{a}} \uplus \mathcal{P} ; \Phi ; t\right) \stackrel{a, \tau}{\longrightarrow} \mathcal{T}_{0}\left(\lfloor P\{x \mapsto u \downarrow\}\rfloor_{a}^{t_{a}} \uplus \mathcal{P} ; \Phi ; t\right) \\
& \text { when } u \downarrow \in \mathcal{T}\left(\Sigma_{c}^{+}, \mathcal{N} \uplus \mathcal{A}\right) \\
& \text { NEW } \quad\left(\lfloor\text { new } n . P\rfloor_{a}^{t_{a}} \uplus \mathcal{P} ; \Phi ; t\right) \stackrel{a, \tau}{\longrightarrow} \mathcal{T}_{0}\left(\left\lfloor P\left\{n \mapsto n^{\prime}\right\}\right\rfloor_{a}^{t_{a}} \uplus \mathcal{P} ; \Phi ; t\right) \\
& \text { with } n^{\prime} \in \mathcal{N} \text { fresh } \\
& \text { IN } \quad\left(\left\lfloor\operatorname{in}^{\star}(x) . P\right\rfloor_{a}^{t_{a}} \uplus \mathcal{P} ; \Phi ; t\right) \stackrel{a, \text { in }^{\star}(u)}{\longrightarrow} \mathcal{T}_{0}\left(\lfloor P\{x \mapsto u\}\rfloor_{a}^{t_{a}} \uplus \mathcal{P} ; \Phi ; t\right)
\end{aligned}
$$

when there exist $b \in \mathcal{A}_{0}$ and $t_{b} \in \mathbb{R}_{+}$such that $t_{b} \leq t-\operatorname{Dist}_{\mathcal{T}_{0}}(b, a)$ and:

- if $b \in \mathcal{A}_{0} \backslash \mathcal{M}_{0}$ then $u \in \operatorname{img}\left(\lfloor\Phi\rfloor_{b}^{t_{b}}\right)$;

- if $b \in \mathcal{M}_{0}$ then $u=R \Phi \downarrow$ for some recipe $R$ such that for all $\mathrm{w} \in \operatorname{vars}(R)$ there exists $c \in \mathcal{A}_{0}$ such that $\mathrm{w} \in \operatorname{dom}\left(\lfloor\Phi\rfloor_{c}^{t_{b}-\operatorname{Dist}_{\tau_{0}}(c, b)}\right)$.

Moreover, in case $\star$ is $<t_{g}$ for some $t_{g}$, we assume in addition that $t_{a}<t_{g}$.

The two first rules are specific to our timed model. The Rst rule allows a process to reset its local clock, whereas the Tim rule allows time to elapse, meaning that all the clocks will be shifted by $\delta$ :

$$
\operatorname{Shift}(\mathcal{P}, \delta)=\biguplus_{\lfloor P\rfloor_{a}^{t_{a}} \in \mathcal{P}} \operatorname{Shift}\left(\lfloor P\rfloor_{a}^{t_{a}}, \delta\right) \text { and } \operatorname{Shift}\left(\lfloor P\rfloor_{a}^{t_{a}}, \delta\right)=\lfloor P\rfloor_{a}^{t_{a}+\delta} .
$$


The remaining rules are quite standard. The Out rule is used to output a message which is immediately added into the frame. The rule LET can be used to apply function symbols, e.g. let $x=\operatorname{dec}(y, k)$ in $P$ applies decryption on top of $y$ with the key $k$ and store the resulting result in $x$ (if this operation succeeds). Otherwise, the process is blocked. This construction is also useful to perform equality tests through the symbol eq as defined in Example 1 and used e.g. in Example 5. The NEw rule allows one to pick a fresh (i.e. previously unused) name. Finally, the IN rule is used to receive a message. One can note the additional side conditions which allows one to model timing constraints: all the messages needed to construct $u$ have to be available to $b$ (who sends $u$ ) at time $t_{b} \leq t-\operatorname{Dist}_{\mathcal{T}_{0}}(b, a)$ to ensure that the message forged and sent by $b$ will have enough time to travel and reach $a$.

Example 8. To illustrate our semantics, we give below a possible execution trace starting from the configuration $K_{0}$ given in Example 7 . We have that:

$$
\begin{aligned}
& K_{0} \stackrel{\left(v_{0}, \tau\right) \cdot\left(v_{0}, \text { out }\left(n_{V}\right)\right)}{\longrightarrow} \mathcal{T}_{0} \rightarrow \mathcal{T}_{0} \stackrel{\left(v_{0}, \text { in }\left(n_{I}\right)\right) \cdot\left(v_{0}, \text { reset }\right) \cdot\left(v_{0}, \tau\right) \cdot\left(v_{0}, \text { out }(c)\right)}{\longrightarrow} \mathcal{T}_{0} \quad\left(\mathcal{P}^{\prime} ; \Phi^{\prime} ; t^{\prime}\right) \\
& \quad \rightarrow \mathcal{T}_{0} \stackrel{\left(v_{0}, \text { in }\left(m_{\text {rep }}\right)\right) \cdot\left(v_{0}, \tau\right) \cdot\left(v_{0}, \tau\right) \cdot\left(v_{0}, \tau\right)}{\longrightarrow} \mathcal{T}_{0} \quad\left(\left\lfloor\mathrm{P}\left(p_{0}, v_{0}\right)\right\rfloor_{p_{0}}^{t^{\prime \prime}} \uplus\left\lfloor\operatorname{end}\left(v_{0}, p_{0}\right)\right\rfloor_{v_{0}}^{\left.t^{\prime \prime}-t^{\prime} ; \Phi^{\prime \prime} ; t^{\prime \prime}\right)}\right.
\end{aligned}
$$

with $m_{\text {rep }}=\operatorname{ans}\left(c, \operatorname{kdf}\left(\operatorname{shk}\left(p_{0}, v_{0}\right), n_{V}, n_{I}\right), \operatorname{shk}\left(p_{0}, v_{0}\right) \oplus \operatorname{kdf}\left(\operatorname{shk}\left(p_{0}, v_{0}\right), n_{V}, n_{I}\right)\right)$, $t^{\prime} \geq \operatorname{Dist}_{\mathcal{T}_{0}}\left(v_{0}, p\right), t^{\prime \prime} \geq 3 \operatorname{Dist}_{\mathcal{T}_{0}}\left(v_{0}, p\right), \Phi^{\prime \prime}=\Phi^{\prime}=\Phi_{0} \uplus\left\{\mathrm{w}_{4} \stackrel{v_{0}, 0}{\longrightarrow} n_{V}, \mathrm{w}_{5} \stackrel{v_{0}, t^{\prime}}{\longrightarrow} c\right\}$.

Here, $n_{I}$ is a name known to the attacker. Formally, we have that $n_{I} \in \Sigma_{0}$. During the first part of the execution $\left(1^{\text {st }}\right.$ line), one instance of the TIM rule has been used. It is necessary to let the verifier receive $n_{I}$. Therefore, we have that $t^{\prime} \geq \operatorname{Dist}_{\mathcal{T}_{0}}\left(v_{0}, p\right)$. The attacker has learnt two messages that have been added into his initial frame $\Phi_{0}$. Then, letting some time to elapse, the process located in $v_{0}$ is able to perform his input action. Indeed, the term $m_{\text {rep }}$ can be forged by $p$ using recipe ans $\left(\mathrm{w}_{5}, R_{0}, R_{1}\right)$ where $R_{0}=\mathrm{kdf}\left(\mathrm{w}_{2} \oplus \mathrm{w}_{3}, \mathrm{w}_{4}, n_{I}\right)$ and $R_{1}=\mathrm{w}_{2} \oplus \mathrm{w}_{3} \oplus R_{0}$. We may note that $m_{\text {rep }}$ passes successfully all the tests, and $v_{0}$ ends his session thinking he is talking to $p_{0}$ (who is actually far away).

\section{Modelling Mafia and Terrorist Frauds}

Here, we aim at proposing a general definition of terrorist fraud in the symbolic setting. Due to its close relationship with the notion of mafia fraud, we first recall how mafia fraud is modelled following the definitions given in [14] before defining the more subtle notion of terrorist fraud.

We start by defining the notion of valid initial configurations which corresponds to the configurations that need to be studied when analysing a given protocol $\mathcal{P}$. Typically, such a configuration will contain instances of the roles of the protocol $\mathcal{P}$ under study.

Definition 2. Let $\mathcal{P}_{\text {prox }}$ be a protocol, $\mathcal{T}_{0}=\left(\mathcal{A}_{0}, \mathcal{M}_{0}\right.$, Loc $\left._{0}, v_{0}, p_{0}\right)$ be a topology, and $\Phi_{0}$ be an initial frame. $K=(\mathcal{P} ; \Phi ; t)$ is a valid initial configuration for $\mathcal{P}_{\text {prox }}$ w.r.t. $\mathcal{T}_{0}$ and $\Phi_{0}$ if $t=0, \Phi=\Phi_{0}$, and for each $\left\lfloor P^{\prime}\right\rfloor_{a^{\prime}}^{t^{\prime}} \in \mathcal{P}$, we have that $t^{\prime}=0$, $a^{\prime} \in \mathcal{A}_{0}$, and either $P^{\prime}=\mathrm{V}\left(a^{\prime}, b^{\prime}\right)$ or $P^{\prime}=\mathrm{P}\left(a^{\prime}, b^{\prime}\right)$ for some $b^{\prime} \in \mathcal{A}_{0}$. 
Now, depending on the type of frauds we consider, the set of topologies under study and the initial knowledge given to the attacker may vary.

\subsection{Mafia fraud}

A mafia fraud is an attack in which generally three agents are involved: a verifier, an honest prover located outside the neighbourhood of the verifier, and an attacker. We consider here its general version which may involve an arbitrary number of participants and we reuse the definition given in [14]. The aim of the attacker is to convince the verifier that the honest prover is actually close to it. The set $\mathcal{C}_{\mathrm{MF}}$ representing all the mafia fraud topologies is given in Example 6 .

Example 9. The topology depicted in Example 7 is a mafia fraud topology. Some other mafia fraud topologies that will be considered later on are depicted below:


The initial knowledge $\Phi_{0}$ we use for defining our initial configuration depends on the topology but it is reasonable to assume that this knowledge is uniform. Therefore, we assume that the initial knowledge of all the participants is given through a template $\mathcal{I}_{0}$, i.e. a set of terms in $\mathcal{T}\left(\Sigma_{c}^{+},\left\{z_{0}, z_{1}\right\}\right)$. Relying on $\mathcal{I}_{0}$, and considering a set $\mathcal{A}_{0}$ of agents, the initial knowledge of agent $a \in \mathcal{A}_{0}$ is given by:

$$
\operatorname{Knows}_{\mathcal{I}_{0}}\left(a, \mathcal{A}_{0}\right)=\left\{u_{0}\left\{z_{0} \mapsto a, z_{1} \mapsto b\right\} \mid u_{0} \in \mathcal{I}_{0} \text { and } b \in \mathcal{A}_{0}\right\}
$$

Given $\mathcal{T}=\left(\mathcal{A}_{0}, \mathcal{M}_{0}\right.$, Loc $\left._{0}, v_{0}, p_{0}\right)$, we denote $\Phi_{\mathcal{I}_{0}}^{\mathcal{T}}$ the initial frame such that $\left\lfloor\operatorname{img}\left(\Phi_{\mathcal{I}_{0}}^{\mathcal{T}}\right)\right\rfloor_{a}^{0}=\operatorname{Knows}_{\mathcal{I}_{0}}\left(a, \mathcal{A}_{0}\right)$ when $a \in \mathcal{M}_{0}$, and $\left\lfloor i m g\left(\Phi_{\mathcal{I}_{0}}^{\mathcal{T}}\right)\right\rfloor_{a}^{0}=\emptyset$ otherwise. Up to a renaming of the handles and some duplicates, $\Phi_{\mathcal{I}_{0}}^{\mathcal{T}}$ is uniquely defined.

Example 10. Continuing our running example, and considering the topology $\mathcal{T}_{\mathrm{MF}}^{0}=\left(\mathcal{A}_{\mathrm{MF}}^{0}, \mathcal{M}_{\mathrm{MF}}^{0}\right.$, Loc $\left._{\mathrm{MF}}^{0}, v_{0}, p_{0}\right)$ (see Example 9), a typical template to derive the initial knowledge of the malicious agents is $\mathcal{I}_{0}=\left\{\operatorname{shk}\left(z_{0}, z_{1}\right)\right.$, $\left.\operatorname{shk}\left(z_{1}, z_{0}\right)\right\}$. Thus, considering the malicious agent $e_{i}$, the set $\operatorname{Knows}_{\mathcal{I}_{0}}\left(e_{i}, \mathcal{A}_{\mathrm{MF}}^{0}\right)$ will contain all the symmetric keys this malicious agent shares with other agents.

When analysing the protocol considering $\mathcal{T}_{\mathrm{MF}}^{0}$, we will consider an initial frame $\Phi_{0}$ containing shk $(a, b)$ for $(a, b) \in\left(\mathcal{A}_{\mathrm{MF}}^{0} \times \mathcal{A}_{\mathrm{MF}}^{0}\right) \backslash\left\{\left(v_{0}, p_{0}\right),\left(p_{0}, v_{0}\right)\right\}$.

Definition 3. Let $\mathcal{P}_{\text {prox }}$ be a protocol and $\mathcal{I}_{0}$ be a template. We say that $\mathcal{P}_{\text {prox }}$ admits a mafia fraud w.r.t. $t_{0}$-proximity if there exist $\mathcal{T} \in \mathcal{C}_{\mathrm{MF}}$, and a valid initial configuration $K$ for $\mathcal{P}_{\text {prox }}$ w.r.t. $\mathcal{T}$ and $\Phi_{\mathcal{I}_{0}}^{\mathcal{T}}$ such that:

$$
K \rightarrow_{\mathcal{T}}^{*}\left(\left\lfloor\operatorname{end}\left(v_{0}, p_{0}\right)\right\rfloor_{a}^{t_{a}} \uplus \mathcal{P} ; \Phi ; t\right) \text { with } \operatorname{Dist}_{\mathcal{T}}\left(v_{0}, p_{0}\right) \geq t_{0}
$$

where $\mathcal{T}=\left(\mathcal{A}_{0}, \mathcal{M}_{0}, \operatorname{Loc}_{0}, v_{0}, p_{0}\right)$. 


\subsection{Terrorist fraud}

Modelling terrorist fraud is tricky. We have to look for a semi-dishonest prover who colludes with the attacker with the aim of letting him authenticate exactly once. We will model this in two steps. First, we will consider all the possible behaviours for this semi-dishonest prover that will allow the attacker to authenticate at least once. Then, to be terrorist fraud resistant, we have to check that any of these behaviours will allow the attacker to re-authenticate later on.

In order to collude with the attacker, one possibility for the prover is to leak his credentials but it is in general not the only option. To define this notion, we consider a simple scenario where $p_{0}$ wants to authenticate to the far away verifier $v_{0}$ through the help of the attacker $a$ located in the neighbourhood of $v_{0}$. This corresponds to the topology $\mathcal{T}_{\text {simple }}^{0}$ given in Example 9.

Definition 4. Let $\mathcal{P}_{\text {prox }}$ be a protocol and $t_{0} \in \mathbb{R}_{+}$. A semi-dishonest prover for $\mathcal{P}_{\text {prox }}$ w.r.t. $t_{0}$ is a process $P_{\text {sd }}$ together with an initial frame $\Phi_{\text {sd }}$ such that:

$$
\left(\left\{\left\lfloor\mathrm{V}\left(v_{0}, p_{0}\right)\right\rfloor_{v_{0}}^{0} ;\left\lfloor P_{\text {sd }}\right\rfloor_{p_{0}}^{0}\right\} ; \emptyset ; 0\right) \stackrel{\text { tr }}{\rightarrow} \mathcal{T}_{\text {simple }}^{0}\left(\left\{\left\lfloor\text { end }\left(v_{0}, p_{0}\right)\right\rfloor_{v_{0}}^{t_{v}} ;\lfloor 0\rfloor p_{p_{0}}^{t_{p}}\right\} ; \Phi ; t\right)
$$

for some $t, t_{v}, t_{p}$, and $\Phi$ such that $\Phi$ and $\Phi_{\text {sd }}$ coincide up to their timestamps.

Note that a semi-dishonest prover can be completely dishonest in the sense that he may leak all his credentials. However, such a semi-dishonest prover can not be honest, i.e. equal to the role of the prover as indicated by the protocol. Indeed, $p_{0}$ is located far away and has to authenticate. Thus, unless the protocol is very bad, the help of the attacker who is close to the verifier will be essential.

Example 11. Going back to our running example, some semi-dishonest provers with their frame are $\left(k=\operatorname{shk}\left(v_{0}, p_{0}\right), m_{0}=\operatorname{kdf}\left(k, n_{V}^{0}, n_{P}^{0}\right)\right.$, and $\left.m_{1}=k \oplus m_{0}\right)$ :

$$
\begin{aligned}
& \text { 1. } P_{\text {sd }}^{1}:=\operatorname{out}(k) \text { with } \Phi_{\text {sd }}^{1}=\left\{\mathrm{w}_{1} \stackrel{v_{0}, 0}{\longrightarrow} n_{V}, \mathrm{w}_{2} \stackrel{p_{0}, 0}{\longrightarrow} k, \mathrm{w}_{3} \stackrel{v_{0}, 0}{\longrightarrow} c\right\} \text {; } \\
& \text { 2. } P_{\text {sd }}^{2}:=\text { new } n_{P} \text {. } \operatorname{in}\left(y_{N}\right) \text {.out }\left(n_{P}\right) \text {. let } y_{0}=\operatorname{kdf}\left(k, y_{N}, n_{P}\right) \text { in } \\
& \text { let } y_{1}=k \oplus y_{0} \text { in out }\left(y_{0}\right) \text {.out }\left(y_{1}\right) \text {. } \\
& \operatorname{in}\left(y_{c}\right) \text {.out }\left(\operatorname{ans}\left(y_{c}, y_{0}, y_{1}\right)\right) \\
& \text { with } \Phi_{\text {sd }}^{2}=\left\{\mathrm{w}_{1} \stackrel{v_{0}, 0}{\longrightarrow} n_{V}, \mathrm{w}_{2} \stackrel{p_{0}, 0}{\longrightarrow} n_{P}, \mathrm{w}_{3} \stackrel{p_{0}, 0}{\longrightarrow} m_{0}, \mathrm{w}_{4} \stackrel{p_{0}, 0}{\longrightarrow} m_{1}, \mathrm{w}_{5} \stackrel{v_{0}, 0}{\longrightarrow} c\right. \text {, } \\
& \left.\mathrm{w}_{6} \stackrel{p_{0}, 0}{\longrightarrow} \text { ans }\left(c, m_{0}, m_{1}\right)\right\} \text {. }
\end{aligned}
$$

The first one actually reveals all his credential to the attacker, and thus the attacker will be able to authenticate later on. The second one reveals less information to the attacker. This is still enough to authenticate once and we will see that this is actually also enough to authenticate later on (see Example 12).

We are now able to define our notion of terrorist fraud resistance. Intuitively, if the dishonest prover gives to his accomplice enough information to pass authentication once, then he will be able to authenticate again without his help.

Definition 5. Let $\mathcal{P}_{\text {prox }}$ be a protocol and $\mathcal{I}_{0}$ be a template. We say that $\mathcal{P}_{\text {prox }}$ is terrorist fraud resistant w.r.t. $t_{0}$-proximity if for all semi-dishonest prover $P_{\mathrm{sd}}$ 
with frame $\Phi_{\text {sd }}$, there exist $\mathcal{T} \in \mathcal{C}_{\mathrm{MF}}$, a valid initial configuration $K$ for $\mathcal{P}_{\text {prox }}$ w.r.t. $\mathcal{T}$ and $\Phi_{\mathcal{I}_{0}}^{\mathcal{T}} \cup \Phi_{\text {sd }}$ such that:

$$
K \stackrel{\text { tr }}{\rightarrow} \mathcal{T}\left(\left\lfloor\text { end }\left(v_{0}, p_{0}\right)\right\rfloor_{v_{0}}^{t^{\prime}} \uplus \mathcal{P} ; \Phi ; t\right) \text { with } \operatorname{Dist}_{\mathcal{T}}\left(v_{0}, p_{0}\right) \geq t_{0}
$$

where $\mathcal{T}=\left(\mathcal{A}_{0}, \mathcal{M}_{0}\right.$, Loc $\left._{0}, v_{0}, p_{0}\right)$.

Example 12. Going back to our running example, we have seen (see Example 8):

$$
K_{0} \stackrel{\operatorname{tr}}{\rightarrow} \mathcal{T}^{0}\left(\left\lfloor\text { end }\left(v_{0}, p_{0}\right)\right\rfloor v_{v_{0}}^{t^{\prime}} \uplus \mathcal{P} ; \Phi ; t\right) .
$$

This execution witnesses the fact that the dishonest prover $P_{\text {sd }}^{2}$ together with frame $\Phi_{\text {sd }}^{2}$ gives enough information to the attacker to allow him to authenticate

later on. This does not mean that the protocol is terrorist fraud resistant since we only consider one particular semi-dishonest prover. To be terrorist fraud resistant, the property has to hold for any semi-dishonest prover.

In our setting, we have the following relationship between mafia fraud and terrorist fraud resistance

Proposition 1. Let $\mathcal{P}_{\text {prox }}$ be a protocol and $\mathcal{I}_{0}$ be a template. If $\mathcal{P}_{\text {prox }}$ admits a mafia fraud then $\mathcal{P}_{\text {prox }}$ is terrorist fraud resistant (w.r.t. $t_{0}$-proximity).

Indeed, whatever the distant semi-dishonest prover discloses (even no information at all), an attacker can still carry out the existing mafia fraud and reauthenticate, therefore impersonating the semi-dishonest prover again. In computational definitions, probability plays a role. In such a setting, a terrorist fraud exists when the semi-dishonest prover can help the attacker to maximise his attack success probability without giving him any advantage for future attacks. The fact that a mafia fraud already exists (with probability 1 in our setting) means that no help can improve the success probability for future attacks, and thus the protocol is terrorist fraud resistant. We may note that distancebounding protocols designed to achieve terrorist fraud resistance aim also to resist against mafia fraud, thus in general achieving terrorist fraud resistance making the protocol vulnerable to a mafia fraud is not an interesting option.

\subsection{Related works}

Up to our knowledge, the only existing definitions of terrorist fraud resistance in the symbolic setting are the one proposed by Chothia et al in [11] and the recent one proposed by Mauw et al in [24].

Chothia et al. Their notion of terrorist fraud is not modelled in two steps as we proposed. Instead, they consider a notion of terrorist prover. Such a process will perform operations on behalf of the attacker, e.g. encrypting and decrypting any values the attacker wishes, but it will never (at least directly) reveal his secrets. Their notion of terrorist prover is appealing but they do not explain how to write such a process. We think that writing such a process is not that easy. 
Example 13. Consider a protocol relying on a hash function $\mathrm{h}$ and we assume that the terrorist prover holds a secret key $k$. A legitimate help that the terrorist prover may give to the attacker without leaking his secret key would consist in computing the hash value of a public data together with his secret key $k$. Therefore, the terrorist prover should contain the oracle: in $(x)$.out $(\mathrm{h}(\langle k, x\rangle))$. In the same spirit, we could argue that $\operatorname{in}(x)$.out $(\mathrm{h}(\langle x, k\rangle))$ is also useful, and perhaps also $\operatorname{in}\left(x_{1}\right) \cdot \operatorname{in}\left(x_{2}\right)$.out $\left(\mathrm{h}\left(\left\langle x_{1},\left\langle k, x_{2}\right\rangle\right\rangle\right)\right)$, etc. Iterating such a reasoning, we do not see how to write a finite terrorist prover that will provide all the valuable help his accomplice may need.

Moreover, when considering a protocol involving an operator with some algebraic properties, e.g. exclusive-or, it seems difficult (perhaps even impossible) to ensure that the terrorist prover will not reveal secrets indirectly).

Example 14. To illustrate this issue, we consider a specific primitive modelled using the equation $\mathrm{g}\left(\mathbf{f}_{1}(x, y), \mathbf{f}_{2}(x, y)\right)=y$. The functions $\mathbf{f}_{1}$ and $\mathbf{f}_{2}$ are two constructor symbols whereas $g$ is a destructor symbol. Following the idea developed in [11], the terrorist prover should contain in $(x)$.out $\left(\mathrm{f}_{1}(x, k)\right)$ as well as $\operatorname{in}(x)$.out $\left(\mathrm{f}_{2}(\langle x, k\rangle)\right)$. However, whereas it is legitimate to provide such an help to the attacker, it seems too strong to give him access to these oracles as soon as he will get $\mathrm{f}_{1}(m, k)$ and $\mathrm{f}_{2}\left(m_{2}, k\right)$ for some message $m$. This example clearly shows that, combining two legitimate helps, the attacker may retrieve some secrets. It is therefore not obvious to describe in a syntactic way the help the terrorist prover is willing to provide.

The main advantage of the definition of terrorist fraud proposed by [11] is probably the fact that it is more amenable to automation using existing verification tools. Indeed, even if the choice of terrorist prover mentioned in [11] is quite debatable, it is fixed, and can therefore be given in input to the verification tool.

Mauw et al. They consider a model based on multiset rewriting rules, and their definition of terrorist fraud is more in line with the one we proposed. In particular, their notion of "valid extensions" of a protocol seems to correspond to our notion of semi-dishonest prover. Then, their definition of terrorist fraud quantifies over all the possible "valid extensions" and this renders the automation of the security analysis difficult. Indeed, no existing verification tool is able to handle this quantification. In [24], they simply illustrate their technique on a toy distance bounding protocol. They provide a manual proof explaining how to get rid of this quantification for this particular toy protocol. Our work explains in a more systematic way how to get rid of this quantification, as well as the one regarding the topology. This is explained in Section 4.

\section{Reduction results}

We first establish a result allowing us to focus on a particular topology. Then, we explain how we get rid of the quantification over semi-dishonest provers. 


\subsection{One topology is enough}

This reduction result regarding the topology is a direct consequence of the proof of the reduction result stated in [14] regarding mafia and distance hijacking frauds. The only new issue here is to take care of the initial frame which contains information from the semi-dishonest prover. This reduction results holds in a rather general setting. We simply assume that the protocol under study is executable.

Definition 6. Given a template $\mathcal{I}_{0}=\left\{u_{1}, \ldots, u_{k}\right\}$, a protocol $\mathcal{P}$ is $\mathcal{I}_{0}$-executable if for any term $u$ (resp. $v$ ) occurring in an out or a let construction in $\mathcal{P}$, there exists a recipe $R \in \mathcal{T}\left(\Sigma_{\text {pub }}^{+},\left\{\mathrm{w}_{1}, \ldots, \mathrm{w}_{k}\right\} \uplus \mathcal{N} \uplus \mathcal{X}\right.$ ) such that $u=R \sigma \downarrow$ (resp. $v \downarrow=R \sigma \downarrow)$ where $\sigma=\left\{\mathrm{w}_{1} \mapsto u_{1}, \ldots, \mathrm{w}_{k} \mapsto u_{k}\right\}$.

Example 15. Going back to our running example described in Example 5, we have that both roles are $\mathcal{I}_{0}$-executable considering $\mathcal{I}_{0}=\left\{\operatorname{shk}\left(z_{0}, z_{1}\right)\right.$, shk $\left.\left(z_{1}, z_{0}\right)\right\}$.

We are now able to state our reduction result regarding terrorist fraud.

Theorem 1. Let $\mathcal{P}_{\text {prox }}$ be an $\mathcal{I}_{0}$-executable protocol w.r.t. some template $\mathcal{I}_{0}$. We have that $\mathcal{P}_{\text {prox }}$ is terrorist fraud resistant w.r.t. $t_{0}$-proximity, if and only if, for all semi-dishonest prover $P_{\text {sd }}$ with frame $\Phi_{\text {sd }}$, there exists a valid initial configuration $K$ for $\mathcal{P}_{\text {prox }}$ w.r.t. $\mathcal{T}_{\mathrm{MF}}^{0}$ and $\Phi_{\mathcal{I}_{0}}^{\mathcal{T}_{\mathrm{MF}}^{0}} \cup \Phi_{\text {sd }}$ such that:

$$
K \stackrel{\operatorname{tr}}{\rightarrow} \mathcal{T}_{\mathrm{MF}}^{0}\left(\left\lfloor\text { end }\left(v_{0}, p_{0}\right)\right\rfloor_{v_{0}}^{t^{\prime}} \uplus \mathcal{P} ; \Phi ; t\right) .
$$

In other words, when analysing terrorist fraud, it is sufficient to consider one particular topology, namely $\mathcal{T}_{\mathrm{MF}}^{0}$ (see Example 9). The key idea to establish the direct part of the theorem consists in showing that behaviours of agents other than $p_{0}$ and $v_{0}$ can be performed by processes executed by malicious agents, and can even be discarded relying on the fact that $\mathcal{P}_{\text {prox }}$ is $\mathcal{I}_{0}$-executable. Then, it remains to map any agent names different from $p_{0}$ and $v_{0}$ to $e_{1}$, and to show that the resulting trace remains executable.

\subsection{One semi-dishonest prover behaviour is enough}

Our second reduction result allows us to focus on a particular semi-dishonest prover when performing our analysis. This results only holds under some hypotheses that are gathered below. We have to rely on the notion of being quasifree for a symbol: $f \in \Sigma_{c}$ is quasi-free if it occurs neither in the equations used to generate the relation $=_{\mathrm{E}}$ nor in the right-hand side of a rewriting rule.

Definition 7. A distance bounding (DB) protocol is a protocol such that:

(i) We have that $\mathrm{V}\left(z_{0}, z_{1}\right)=$ block $_{V}$.reset.new $c$.out $(c) \cdot$ in $^{<2 \times t_{0}}(x) \cdot$ block $_{V}^{\prime}$, and $\mathrm{P}\left(z_{0}, z_{1}\right)=\operatorname{block}_{P} \cdot \operatorname{in}\left(y_{c}\right)$.out $(u) \cdot$ block $_{P}^{\prime}$ where block $_{X}$ and block ${ }_{X}^{\prime}$ with $X \in\{V, P\}$ is a sequence of actions without reset and guarded input instructions. Moreover, we assume that out $(c)$ (resp. in $\left(y_{c}\right)$ ) corresponds to the $i_{0}{ }^{\text {th }}$ communication action of $\mathrm{P}\left(z_{0}, z_{1}\right)$ (resp. $\mathrm{V}\left(z_{0}, z_{1}\right)$ ) for some $i_{0}$. 
(ii)

$$
\begin{aligned}
& \left(\left\lfloor\mathrm{V}\left(v_{0}, p_{0}\right)\right\rfloor_{v_{0}}^{0} \uplus\left\lfloor\mathrm{P}\left(p_{0}, v_{0}\right)\right\rfloor_{p_{0}}^{0} ; \emptyset ; 0\right) \stackrel{\text { tr }}{\rightarrow} \mathcal{T}_{\text {basic }}^{0}\left(\lfloor 0\rfloor_{v_{0}}^{0} \uplus\lfloor 0\rfloor_{p_{0}}^{0} ; \Phi ; 0\right) \text { with } \\
& \operatorname{tr}=\left\{\begin{array}{l}
\left(a_{1}, \text { out }\left(m_{1}\right)\right) \cdot\left(b_{1}, \operatorname{in}\left(m_{1}\right)\right) \ldots\left(a_{i_{0}-1}, \operatorname{out}\left(m_{i_{0}-1}\right)\right) \cdot\left(b_{i_{0}-1}, \operatorname{in}\left(m_{i_{0}-1}\right)\right) \\
\left(v_{0}, \operatorname{out}\left(m_{i_{0}}\right)\right) \cdot\left(p_{0}, \operatorname{in}\left(m_{i_{0}}\right)\right) \cdot\left(p_{0}, \operatorname{out}\left(m_{i_{0}+1}\right)\right) \cdot\left(v_{0}, \operatorname{in}<t\left(m_{i_{0}+1}\right)\right) \\
\left(a_{n-1}, \operatorname{out}\left(m_{n-1}\right) \cdot\left(b_{n-1}, \operatorname{in}\left(m_{n-1}\right)\right) \ldots\left(a_{n}, \text { out }\left(m_{n}\right) \cdot\left(b_{n}, \operatorname{in}\left(m_{n}\right)\right)\right.\right.
\end{array}\right. \\
& \text { up to } \tau \text { actions, and }\left\{a_{i}, b_{i}\right\}=\left\{v_{0}, p_{0}\right\} \text { for any } i \in\{1, \ldots, n\} \backslash\left\{i_{0}, i_{0}+1\right\} .
\end{aligned}
$$

(iii) Let $\mathcal{U}=\left\{x=u \mid\right.$ "let $x=u$ in "occurs in $\left.\mathrm{V}\left(v_{0}, p_{0}\right)\right\}$. We assume that $\operatorname{csu}(\mathcal{U})$ exists and is reduced to a singleton $\left\{\theta_{\mathcal{P}}\right\}$. Moreover, we assume that $\left(x_{1}, \ldots, x_{k}\right) \theta_{\mathcal{P}} \downarrow \sigma=m_{i_{1}}, \ldots, m_{i_{k}}$ where $x_{1}, \ldots, x_{k}$ are the variables occurring in input in the role $\mathrm{V}\left(v_{0}, p_{0}\right), i_{1}, \ldots, i_{k}$ are the indices among $1, \ldots, n$ corresponding to input performed by $v_{0}$, and $\sigma$ is a bijective renaming from variables to names freshly generated by $\mathrm{P}\left(p_{0}, v_{0}\right)$ when executing tr.

(iv) We assume the existence of a context $C$ made of quasi-free public function symbols such that $u=C\left[y_{c}, u_{1}, \ldots, u_{p}\right]$, and $y_{c}$ does not occur in $u_{1}, \ldots, u_{p}$.

The two first conditions put some restrictions on the shape of the roles. In particular, we assume that if no attacker interferes, these two roles together will execute until the end. The third condition gives us the existence of a unique most general unifier (modulo $\mathrm{E}$ ) and is actually satisfied by many term algebra of interest for protocol verification, e.g. the one described in Section 2.1. Actually, any rewriting system with only one rule per destructor will satisfy such an hypothesis. It may seem restrictive that in a normal execution messages that are exchanged have the shape indicated by $\theta_{\mathcal{P}}$ but this requirement is in general always satisfied. Note that otherwise, it would mean that some terms sent by the prover are never entirely checked during the protocol execution, and thus are useless. Condition (iv) allows us to ensure that there exists a strategy for the semi-dishonest prover. This strategy will consist of sending the terms $u_{1}, \ldots, u_{n}$ in advance to his accomplice, and let him to compute (as indicated by $C$ ) the answer to the challenge from $u_{1}, \ldots, u_{n}$ and the challenge $c^{\prime}$ he will receive from the verifier. Actually, the best strategy will consist in considering $C_{\mathcal{P}}$ the smallest context (in terms of number of symbols) satisfying the requirements.

Example 16. Going back to our running example, all the conditions stated in Definition 7, are indeed satisfied. Assuming that names are not renamed when executing NEw, we obtain the following trace:

$$
\operatorname{tr}=\left\{\begin{array}{l}
\left(v_{0}, \operatorname{out}\left(n_{V}\right)\right) \cdot\left(p_{0}, \operatorname{in}\left(n_{V}\right)\right) \cdot\left(p_{0}, \operatorname{out}\left(n_{P}\right)\right) \cdot\left(v_{0}, \operatorname{in}\left(n_{P}\right)\right) \\
\left(v_{0}, \operatorname{out}(c)\right) \cdot\left(p_{0}, \operatorname{in}(c)\right) \cdot\left(p_{0}, \operatorname{out}\left(\operatorname{ans}\left(c, m_{0}, m_{1}\right)\right)\right) \cdot\left(v_{0}, \operatorname{in}\left(\operatorname{ans}\left(c, m_{0}, m_{1}\right)\right)\right)
\end{array}\right.
$$

where $m_{0}=\operatorname{kdf}\left(\operatorname{shk}\left(p_{0}, v_{0}\right), n_{V}, n_{P}\right)$ and $m_{1}=\operatorname{shk}\left(p_{0}, v_{0}\right) \oplus m_{0}$.

Regarding condition (iii), we have that $\theta_{P}$ as defined in Example 4 and $\sigma=\left\{x_{N} \mapsto n_{P}\right\}$ satisfy our requirement. Regarding condition (iv), we have that $u_{1}=y_{0}$, and $u_{2}=y_{1}$, and thus $C_{\mathcal{P}}$ only contains the quasi-free symbol ans.

According to our definition, when analysing a protocol $\mathcal{P}_{\text {prox }}$ w.r.t. terrorist frauds you should consider all the possible semi-dishonest provers. However, for the class of distance bounding protocol we consider, we will show that we can restrict our attention to a particular dishonest prover that we define now. 
Definition 8. Let $\mathcal{P}_{\text {prox }}$ be a DB protocol as given in Definition 7. The most general semi-dishonest prover for $\mathcal{P}_{\text {prox }}$, denoted $\mathrm{P}^{*}$, is the process:

$\left(\operatorname{block}_{P}\right.$.out $\left.\left(u_{1}\right) \ldots \operatorname{out}\left(u_{k}\right) \cdot \operatorname{in}\left(y_{c}\right) \cdot \operatorname{out}(u) \cdot \operatorname{block}_{P}^{\prime}\right)\left\{z_{0} \mapsto p_{0}, z_{1} \mapsto v_{0}\right\}$ where $u_{1}, \ldots, u_{p}$ are the terms such that $u=C_{\mathcal{P}}\left[y_{c}, u_{1}, \ldots, u_{p}\right]$.

Its associated frame, denoted $\Phi^{*}$, is the one obtained considering the normal execution and letting the attacker answer to the challenge relying on $C_{\mathcal{P}}$.

The most general semi-dishonest prover will help his accomplice by sending him (before the rapid phase starts) the material he needs to perform this phase alone. For this, the most general semi-dishonest prover will send messages corresponding to the maximal subterms of $u$ that do not contain the challenge. This will be sufficient to answer to the challenge sent by the verifier, and we will see that this is actually the strategy that leaks the least information.

Example 17. Going back to our running example, $P_{\mathrm{sd}}^{1}$ together with frame $\Phi_{\mathrm{sd}}^{1}$ as described in Example 11 corresponds to the most general semi-dishonest prover.

Note that the most general semi-dishonest prover $\mathrm{P}^{*}$ (as given in Definition 8) is a dishonest prover. This simply means that such a process when put together with $\left\lfloor\mathrm{V}\left(v_{0}, p_{0}\right)\right\rfloor_{v_{0}}^{0}$ can be fully executed considering the topology $\mathcal{T}_{\text {simple }}^{0}$. This is actually an easy consequence of our definition of DB protocol exploiting the fact that $\mathrm{P}^{*}$ and $\mathrm{P}\left(p_{0}, v_{0}\right)$ are rather similar. More interestingly, we can establish a strong relationship between the frame $\Phi^{*}$ (the one associated to $\mathrm{P}^{*}$ ) and a frame $\Phi_{\text {sd }}$ associated to an arbitrary semi-dishonest prover $P_{\text {sd }}$.

Proposition 2. Let $\mathcal{P}_{\text {prox }}$ be a DB protocol, and $\mathrm{P}^{*}$ be its most general semidishonest prover with $\Phi^{*}$ its associated frame. Let exec* be the execution witnessing the fact that $\mathrm{P}^{*}$ together with $\Phi^{*}$ is a semi-dishonest prover, i.e.:

$\operatorname{exec}^{*}:\left(\left\{\left\lfloor\mathrm{V}\left(v_{0}, p_{0}\right)\right\rfloor_{v_{0}}^{0},\left\lfloor\mathrm{P}^{*}\right\rfloor_{p_{0}}^{0}\right\} ; \emptyset ; 0\right) \stackrel{\operatorname{tr}^{*}}{\longrightarrow} \mathcal{T}_{\text {simple }}^{0}\left(\left\{\left\lfloor\operatorname{end}\left(v_{0}, p_{0}\right)\right\rfloor_{v_{0}}^{t_{v}^{*}},\lfloor 0\rfloor_{p_{0}}^{t_{p}^{*}}\right\} ; \Phi^{*} ; t^{*}\right)$.

Let $\mathrm{P}_{\mathrm{sd}}$ be a semi-dishonest prover for $\mathcal{P}_{\text {prox }}$ together with its frame $\Phi_{\mathrm{sd}}$, and exec be the execution witnessing this fact, i.e.

exec: $\left(\left\{\left\lfloor\mathrm{V}\left(v_{0}, p_{0}\right)\right\rfloor_{v_{0}}^{0},\left\lfloor\mathrm{P}_{\text {sd }}\right\rfloor_{p_{0}}^{0}\right\} ; \emptyset ; 0\right) \stackrel{\text { tr }}{\rightarrow} \mathcal{T}_{\text {simple }}^{0}\left(\left\{\left\lfloor\operatorname{end}\left(v_{0}, p_{0}\right)\right\rfloor_{v_{0}}^{t_{v}},\lfloor 0\rfloor_{p_{0}}^{t_{p}}\right\} ; \Phi_{\text {sd }} ; t\right)$.

We have that there exists a substitution $\sigma: \mathcal{N} \rightarrow \mathcal{T}\left(\Sigma_{c}^{+}, \mathcal{N} \cup \mathcal{A}\right)$ from names freshly generated by $\mathrm{P}^{*}$ to constructor terms such that for any out $(u)$ occurring in $\mathrm{tr}^{*}$, there exists a recipe $R$ such that $R \Phi_{\mathrm{sd}} \downarrow={ }_{\mathrm{E}} u \sigma$.

Roughly, up to some substitution $\sigma$, we know that an arbitrary dishonest prover will disclose more information than the general one. Thus, to analyse terrorist fraud resistance, it is sufficient to consider the most general semi-dishonest prover. This actually corresponds to the best strategy for the terrorist prover.

Theorem 2. Let $\mathcal{P}_{\text {prox }}$ be a DB protocol and $\mathcal{I}_{0}$ be a template. Let $\Phi^{*}$ be the frame associated to the most general semi-dishonest prover of $\mathcal{P}_{\text {prox }}$. We have that $\mathcal{P}_{\text {prox }}$ is terrorist fraud resistant w.r.t. $t_{0}$-proximity if, and only if, there exists a 
topology $\mathcal{T}=\left(\mathcal{A}_{0}, \mathcal{M}_{0}\right.$, Loc $\left._{0}, v_{0}, p_{0}\right) \in \mathcal{C}_{\mathrm{MF}}$ and a valid initial configuration $K_{0}$ for $\mathcal{P}_{\text {prox }}$ w.r.t. $\mathcal{T}$ and $\Phi^{*} \cup \Phi_{\mathcal{I}_{0}}^{\mathcal{T}}$ such that:

$$
K_{0} \stackrel{\operatorname{tr}}{\rightarrow}_{\mathcal{T}}\left(\left\lfloor\text { end }\left(v_{0}, p_{0}\right)\right\rfloor v_{v_{0}}^{t^{\prime}} \uplus \mathcal{P} ; \Phi ; t\right) \text { with } \operatorname{Dist}_{\mathcal{T}}\left(v_{0}, p_{0}\right) \geq t_{0} .
$$

We establish this result by showing that an execution trace tr starting with $\Phi^{*} \cup \Phi_{\mathcal{I}_{0}}^{\mathcal{T}}$ as an initial frame can be mimicked by an execution trace tr $\sigma$ starting with the initial frame $\Phi_{\text {sd }} \cup \Phi_{\mathcal{I}_{0}}^{\mathcal{T}}$. In other words, $P_{\text {sd }}$ is not better than $\mathrm{P}^{*}$ : the information leaked by $\Phi_{\text {sd }}$ will also allow the accomplice to authenticate again.

\subsection{Main result}

Applying Theorem 1 to reduce the topology, and then Theorem 2 to narrow down the number of semi-dishonest provers to consider, we get rid of the quantifications over semi-dishonest provers as well as the one regarding the topology. We now state our main reduction result.

Corollary 1. Let $\mathcal{P}_{\text {prox }}$ be a $D B$ protocol and $\mathcal{I}_{0}$ be a template such that $\mathcal{P}$ is $\mathcal{I}_{0}$-executable. Let $\mathrm{P}^{*}$ be the most general semi-dishonest prover for $\mathcal{P}$ together with its associated frame $\Phi^{*}$. We have that $\mathcal{P}_{\text {prox }}$ is terrorist fraud resistant w.r.t. $t_{0}$-proximity if, and only if, there exists a valid initial configuration $K_{0}$ for $\mathcal{P}_{\text {prox }}$ w.r.t. $\mathcal{T}_{\mathrm{MF}}^{0}$ and $\Phi^{*} \cup \Phi_{\mathcal{I}_{0}}^{\mathcal{T}_{\mathrm{MF}}^{0}}$ such that:

$$
K_{0} \stackrel{\operatorname{tr}}{\rightarrow}_{\mathcal{T}_{\mathrm{MF}}^{t_{0}}}\left(\left\lfloor\text { end }\left(v_{0}, p_{0}\right)\right\rfloor{ }_{v_{0}}^{t^{\prime}} \uplus \mathcal{P} ; \Phi ; t\right) .
$$

We will see in the next section that our definition of DB protocol is quite general and covers most existing distance bounding protocols. However, some existing protocols, like Brands \& Chaum [8] and MAD [10], do not qualify for our approach. The former does not satisfy our hypothesis (iv) whereas the latter starts with a commit on the challenge value, preventing it to be fresh (hypothesis (i) is not satisfied). Despite this, since these two protocols are subject to a terrorist fraud, we could simply exhibit the corresponding semi-dishonest prover and use our methodology to establish the existence a terrorist fraud.

A reduction result allowing one to get rid of the quantification over semidishonest provers is also suggested in [24]. The reduction result is not formally stated. Instead, the authors provide a manual proof of resistance to terrorist fraud for a specific DBToy protocol relying on the idea of least-disclosing message. Then, the authors claim that similar proofs can be done on all the case studies they have looked at. We would like to emphasise that even if our conditions (expressed in Definition 7) are not necessarily tight, the freshness of the challenge just as the rapid phase starts is necessary to ensure the completeness of our approach. Otherwise, a best strategy for the semi-dishonest prover could be to send out a message which contains the challenge. This condition is missing in [24] and therefore their approach is not complete even for protocols satisfying their least-disclosing message assumption. 


\begin{tabular}{l|cc||l|cc|} 
Protocols & MFR & TFR & Protocols & MFR & TFR \\
\hline Basin's Toy Example [4] & $\checkmark$ & $\checkmark$ & Swiss-Knife [22] & $\checkmark$ & $\checkmark$ \\
Hancke and Kuhn [20] & $\checkmark$ & $\times$ & Modified Swiss-Knife [18] & $\checkmark$ & $\times$ \\
Modified Hancke and Kuhn [29] & $\checkmark$ & $\checkmark$ & Munilla et al. [27] & $\checkmark$ & $\times$ \\
TREAD-PKey V1 [3] & $\times$ & $\checkmark$ & SPADE [9] & $\times$ & $\checkmark$ \\
TREAD-PKey V1 Fixed [19] & $\checkmark$ & $\checkmark$ & SPADE Fixed [19] & $\checkmark$ & $\checkmark$ \\
TREAD-PKey V2 [3] & $\times$ & $\checkmark$ & SKI [7] & $\checkmark$ & $\checkmark$ \\
TREAD-PKey V2 Fixed [19] & $\checkmark$ & $\checkmark$ & PaySafe [12] & $\checkmark$ & $\times$ \\
TREAD-SKey [3] & $\checkmark$ & $\checkmark$ & NXP [21] & $\checkmark$ & $\times$
\end{tabular}

Table 1: Results on our case studies ( $\times$ : attack found, $\checkmark$ : proved secure)

\section{Case studies}

Getting rid of the quantifications, we apply techniques already used in e.g. [12, 14], to leverage the verification tool ProVerif to analyse terrorist fraud on distance bounding protocols.

\subsection{Analysing terrorist-fraud resistance using Proverif}

Based on the technique described in [14], we reuse the syntax of phases included in Proverif to model the guarded input of a Verifier. We will consider the same transformation, while adding an extra phase at the beginning (phase 0) to enrich the knowledge of the attacker with the frame provided together with the mostgeneral semi-dishonest prover. Then, we consider a Verifier-Test modelled using three phases (1,2 and 3) to see whether the adversary can re-authenticate itself by impersonating the Prover or not. As in [14], we also give to the adversary the possibility to play with all the agents present in the topology if they are close enough, since they can provide useful information.

Depending on Proverif outputs, we conclude on the terrorist-fraud security of the distance-bounding protocol. Either it is not possible to reach the end event of the Verifier-Test, or the tool returns a trace in which the event is reachable. In the first case, the attacker can not authenticate itself to the Verifier, even with the help provided by the Prover in phase 0, meaning that the protocol is vulnerable to a terrorist-fraud attack. In the second case, we first need to ensure that the trace provided by ProVerif is a valid trace in our model. If this is the case, then the adversary can authenticate itself to the Verifier again, without further help from the Prover, meaning that the protocol is terroristfraud resistant. Note that, even if in theory, our approach may not allow one to conclude (in case e.g. ProVerif does not terminate or simply say cannot be proved), we never encountered this situation when performing our case studies.

\subsection{Our results}

We applied this methodology to different well-known distance-bounding protocols as long as they met the hypotheses needed by our approach, as mentioned 


$$
\begin{aligned}
& \operatorname{Verifier}\left(s k_{V}, p k_{P}\right) \quad \operatorname{Prover}\left(s k_{P}, p k_{V}\right) \\
& \text { new } \quad\left\{b, \mathbf{V}, \sigma_{P}\right\}_{p k_{V}} \quad \text { new } \\
& \text { m, } a \quad \leftarrow-\cdots \quad b
\end{aligned}
$$

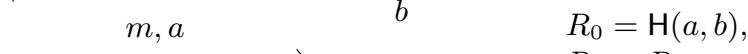

$$
\begin{aligned}
& \begin{array}{cc}
c & \text { new } \\
& r=\operatorname{Ans}\left(c, R_{0}, R_{1}\right)
\end{array} \\
& R_{1}=R_{0} \oplus m \oplus b \text {, and } \\
& \sigma_{P}=\operatorname{Sign}_{s k_{P}}(b, \mathbf{V}) \\
& \mathrm{H}(a, b, m, c, r)
\end{aligned}
$$

Fig. 1: Description of the SPADE (fixed) protocol

in Section 4. As expected, numerous existing protocols qualify and the results are shown in Table 1. All our implementation files can be found in [15]. The tool concludes in less than a minute for most of the examples, except for two protocols: SPADE and SKI, where the extensive use of the xor operator may explain this noteworthy difference. To comply with the use of the symbolic approach, we needed to replace the actual bit-sized rapid exchanges by a single round of challenge-response using one fresh nonce, as presented in the examples throughout this paper. Moreover, due to Proverif limitations, we only considered, when needed, a weak version of the xor operator.

Our results confirm existing mafia frauds against the SPADE and the TREAD (PKey version) protocols $[14,23]$. Therefore we considered the fixed versions of these protocols mentioned in [19] and proved them mafia-fraud and terroristfraud resistant using our methodology. The fix, which consists in adding the Verifier identity in the first message sent by the Prover, is illustrated for the SPADE protocol in the Figure 1 above.

We also extended our analysis to contactless payment protocols, e.g. Paysafe and NXP. While it was not surprising that they do not offer terrorist-fraud resistance, we consider that those protocols should claim if they want to support such a security property or not. Indeed, allowing terrorist fraud could be a feature of the card, permitting its user to agree for a one-time payment to a third-party while not being physically next to it, without risking any non-expected following payment, similarly to the current virtual credit card system.

\subsection{Limitations}

Even if our methodology is general enough to deal with a number of examples, we had to cope with some limitations. First, coming from the tool, Proverif, as mentioned earlier, we needed to weaken the xor operator. While our methodology could consider a different tool which deals better with such an operator, like Tamarin [26], it appears that Tamarin also behaves poorly depending on the considered protocols. Indeed, we faced up with non-termination issues when we tried to apply our methodology to the Brands and Chaum protocol within Tamarin. These termination issues are also visible in the case studies performed by Mauw et al in [23] (using Tamarin) in which they often had to weaken the xor operator. 
Second, as already discussed in 4.3, some limitations are due to the hypotheses we need on a distance-bounding protocols to conduct our formal development. We believe that we could relax our hypothesis regarding the freshness of the challenge up to a non-deductibility hypothesis to be able to apply our methodology to a protocol like MAD, but this is left to future work.

\section{References}

1. M. Abadi and C. Fournet. Mobile values, new names, and secure communication. In Proc. 28th Symposium on Principles of Programming Languages (POPL'01), pages 104-115. ACM Press, 2001.

2. G. Avoine, M. A. Bingöl, S. Kardas, C. Lauradoux, and B. Martin. A framework for analyzing RFID distance bounding protocols. Journal of Computer Security, 19(2):289-317, 2011.

3. G. Avoine, X. Bultel, S. Gambs, D. Gerault, P. Lafourcade, C. Onete, and J.M. Robert. A terrorist-fraud resistant and extractor-free anonymous distancebounding protocol. In Proc. 12th ACM Asia Conference on Computer and Communications Security. ACM Press, 2017.

4. D. Basin, S. Capkun, P. Schaller, and B. Schmidt. Formal reasoning about physical properties of security protocols. ACM Transactions on Information and System Security (TISSEC), 14(2):16, 2011.

5. B. Blanchet. An Efficient Cryptographic Protocol Verifier Based on Prolog Rules. In Proc. 14th Computer Security Foundations Workshop (CSFW'01), pages 82-96. IEEE Computer Society Press, 2001.

6. B. Blanchet. Modeling and verifying security protocols with the applied pi calculus and proverif. Foundations and Trends in Privacy and Security, 1(1-2):1-135, 2016.

7. I. Boureanu, A. Mitrokotsa, and S. Vaudenay. Secure and lightweight distancebounding. In International Workshop on Lightweight Cryptography for Security and Privacy, pages 97-113. Springer, 2013.

8. S. Brands and D. Chaum. Distance-bounding protocols. In Workshop on the Theory and Application of of Cryptographic Techniques, pages 344-359. Springer, 1993.

9. X. Bultel, S. Gambs, D. Gerault, P. Lafourcade, C. Onete, and J. Robert. A prover-anonymous and terrorist-fraud resistant distance-bounding protocol. In Proc. 9th ACM Conference on Security $\&$ Privacy in Wireless and Mobile Networks, (WISEC'16), pages 121-133. ACM Press, 2016.

10. S. Čapkun, L. Buttyán, and J.-P. Hubaux. Sector: secure tracking of node encounters in multi-hop wireless networks. In Proc. 1st ACM workshop on Security of ad hoc and sensor networks, pages 21-32. ACM, 2003.

11. T. Chothia, J. de Ruiter, and B. Smyth. Modelling and analysis of a hierarchy of distance bounding attacks. In Proc. 27th USENIX Security Symposium, USENIX Security 2018, 2018.

12. T. Chothia, F. D. Garcia, J. de Ruiter, J. van den Breekel, and M. Thompson. Relay cost bounding for contactless EMV payments. In Proc. 19th International Conference on Financial Cryptography and Data Security (FC'15), volume 8975 of LNCS. Springer, 2015.

13. A. Debant and S. Delaune. Symbolic verification of distance bounding protocols. In Proc. 8th International Conference on Principles of Security and Trust (POST'19), LNCS. Springer, 2019. 
14. A. Debant, S. Delaune, and C. Wiedling. A symbolic framework to analyse physical proximity in security protocols. In Proc. 38th IARCS Annual Conference on Foundations of Software Technology and Theoretical Computer Science, (FSTTCS'18), volume 122 of LIPIcs. Schloss Dagstuhl - Leibniz-Zentrum fuer Informatik, 2018.

15. A. Debant, S. Delaune, and C. Wiedling. Symbolic Analysis of Terrorist Fraud Resistance. Research report, Univ Rennes, CNRS, IRISA, France, July 2019.

16. D. Dolev and A. Yao. On the security of public key protocols. IEEE Transactions on information theory, 29(2):198-208, 1983.

17. U. Dürholz, M. Fischlin, M. Kasper, and C. Onete. A formal approach to distancebounding RFID protocols. In Proc. 14th International Conference on Information Security (ISC'11), volume 7001 of LNCS. Springer, 2011.

18. M. Fischlin and C. Onete. Terrorism in distance bounding: Modeling terroristfraud resistance. In Proc. 11th International Conference on Applied Cryptography and Network Security (ACNS'13), volume 7954 of LNCS. Springer, 2013.

19. D. Gerault. Security Analysis of Contactless Communication Protocols. PhD thesis, Université Clermont Auvergne, 2018.

20. G. P. Hancke and M. G. Kuhn. An RFID distance bounding protocol. In Proc. 1st International Conference on Security and Privacy for Emerging Areas in Communications Networks (SECURECOMM'05), pages 67-73. IEEE, 2005.

21. P. Janssens. Proximity check for communication devices, Oct. 31 2017. US Patent $9,805,228$.

22. C. H. Kim, G. Avoine, F. Koeune, F. Standaert, and O. Pereira. The SwissKnife RFID distance bounding protocol. In Proc. 11th International Conference on Information Security and Cryptology (ICISC'08), volume 5461 of LNCS. Springer, 2008.

23. S. Mauw, Z. Smith, J. Toro-Pozo, and R. Trujillo-Rasua. Distance-bounding protocols: Verification without time and location. In Proc. 39th IEEE Symposium on Security and Privacy (SEP'18), pages 152-169, 2018.

24. S. Mauw, Z. Smith, J. Toro-Pozo, and R. Trujillo-Rasua. Post-Collusion Security and Distance Bounding. In (To appear in) Proceedings of the 2019 ACM SIGSAC Conference on Computer and Communications Security. ACM, 2019.

25. C. Meadows, R. Poovendran, D. Pavlovic, L. Chang, and P. Syverson. Distance bounding protocols: Authentication logic analysis and collusion attacks. In $\mathrm{Se}$ cure localization and time synchronization for wireless sensor and ad hoc networks, pages 279-298. Springer, 2007.

26. S. Meier, B. Schmidt, C. Cremers, and D. Basin. The Tamarin Prover for the Symbolic Analysis of Security Protocols. In Proc. 25th International Conference on Computer Aided Verification (CAV'13), volume 8044 of LNCS, pages 696-701. Springer, 2013.

27. J. Munilla and A. Peinado. Distance bounding protocols for rfid enhanced by using void-challenges and analysis in noisy channels. Wireless communications and mobile computing, 8(9):1227-1232, 2008.

28. V. Nigam, C. Talcott, and A. A. Urquiza. Towards the automated verification of cyber-physical security protocols: Bounding the number of timed intruders. In Proc. 21st European Symposium on Research in Computer Security (ESORICS'16), pages 450-470. Springer, 2016.

29. S. Vaudenay. On modeling terrorist frauds - addressing collusion in distance bounding protocols. In Proc. 7th International Conference on Provable Security (ProvSec'13), volume 8209 of $L N C S$, pages 1-20. Springer, 2013.

30. S. Vaudenay, I. Boureanu, A. Mitrokotsa, et al. Practical \& provably secure distance-bounding. In Proc. 16th Information Security Conference (ISC'13), 2013. 


\section{A Proofs of our results}

Proposition 1. Let $\mathcal{P}_{\text {prox }}$ be a protocol and $\mathcal{I}_{0}$ be a template. If $\mathcal{P}_{\text {prox }}$ admits a mafia fraud then $\mathcal{P}_{\text {prox }}$ is terrorist fraud resistant (w.r.t. $t_{0}$-proximity).

Proof. If $\mathcal{P}_{\text {prox }}$ admits a mafia fraud w.r.t. $t_{0}$-proximity then there exists a topology $\mathcal{T}=\left(\mathcal{A}_{0}, \mathcal{M}_{0}\right.$, $\left.\operatorname{Loc}_{0}, v_{0}, p_{0}\right) \in \mathcal{C}_{\mathrm{MF}}$ and a valid initial configuration $K=\left(\mathcal{P}_{0} ; \Phi_{0} ; 0\right)$ for $\mathcal{P}_{\text {prox }}$ w.r.t. $\mathcal{T}$ and $\Phi_{\mathcal{I}_{0}}^{\mathcal{T}}$ such that:

$$
K \rightarrow_{\mathcal{T}}^{*}\left(\left\lfloor\operatorname{end}\left(v_{0}, p_{0}\right)\right\rfloor_{v_{0}}^{t_{v}} \uplus \mathcal{P} ; \Phi_{0} \cup \Phi ; t\right) \text { with } \operatorname{Dist}_{\mathcal{T}}\left(v_{0}, p_{0}\right) \geq t_{0}
$$

for some multiset of extended processes $\mathcal{P}$, frame $\Phi$ and times $t_{v}, t \in \mathbb{R}_{+}$.

For all $\Phi_{\text {sd }}$ together with a frame $\Phi_{\text {sd }}$ a semi-dishonest prover for $\mathcal{P}_{\text {prox }}$, we have that $K^{\prime}=\left(\mathcal{P}_{0} ; \Phi_{0} \cup \Phi_{\text {sd }} ; 0\right)$ is a valid initial configuration for $\mathcal{P}_{\text {prox }}$ w.r.t. $\mathcal{T}$ and $\Phi_{\mathcal{I}_{0}}^{\mathcal{T}} \cup \Phi_{\text {sd }}$. The same execution applies since the initial knowledge of the attacker has just been increased.

We can thus conclude that $\mathcal{P}_{\text {prox }}$ is terrorist fraud resistant.

\section{A.1 Proofs of Section 4.1}

This reduction result is very close to the one stated and proved in [14] for mafia fraud. However, we have to take care of the extra messages added into the frame that may contain some agent names (other than $v_{0}, p_{0}, e_{1}$, and $e_{2}$ ). Actually, the transformation applied to reduce the topology will modify these names. Thus, to make sure that the reduction result given in [14] applies, we will first show that names other than $v_{0}, p_{0}, e_{1}$, and $e_{2}$ are not mandatory in the initial frame.

Lemma 1. Let $\mathcal{P}_{\text {prox }}$ be an $\mathcal{I}_{0}$-executable protocol w.r.t. some template $\mathcal{I}_{0}$. Moreover, we assume that there exists a valid initial configuration for $\mathcal{P}_{\text {prox }}$ w.r.t. $\mathcal{T}_{\mathrm{MF}}^{0}$ and $\Phi_{\mathcal{I}_{0}}^{\mathcal{T}_{\mathrm{MF}}^{0}} \cup \Phi_{\mathrm{sd}}$ such that $K \stackrel{\mathrm{tr}}{\rightarrow} \mathcal{T}_{\mathrm{MF}}^{0}\left(\left\lfloor\text { end }\left(v_{0}, p_{0}\right)\right\rfloor_{v_{0}}^{t_{v}} \uplus \mathcal{P} ; \Phi ; t\right)$ for any semidishonest prover $P_{\text {sd }}$ with frame $\Phi_{\text {sd }}$ such that names $\left(\Phi_{\text {sd }}\right) \cap \mathcal{A} \subseteq\left\{v_{0}, p_{0}, e_{1}, e_{2}\right\}$.

Let $P_{\mathrm{sd}}^{\prime}$ be a semi-dishonest prover with frame $\Phi_{\mathrm{sd}}^{\prime}$. We have that there exists a valid initial configuration $K^{\prime}$ for $\mathcal{P}_{\text {prox }}$ w.r.t. $\mathcal{T}_{\mathrm{MF}}^{0}$ and $\Phi_{\mathcal{I}_{0}}^{\mathcal{T}_{\mathrm{MF}}^{0}} \cup \Phi_{\text {sd }}^{\prime}$ such that

$$
K^{\prime} \stackrel{\operatorname{tr}^{\prime}}{\rightarrow} \mathcal{T}_{\mathrm{MF}}^{\mathrm{O}}\left(\left\lfloor\operatorname{end}\left(v_{0}, p_{0}\right)\right\rfloor_{v_{0}}^{t_{v}^{\prime}} \uplus \mathcal{P}^{\prime} ; \Phi^{\prime} ; t^{\prime}\right) .
$$

Proof. We consider $P_{\text {sd }}^{\prime}$ a semi-dishonest prover with frame $\Phi_{\text {sd }}^{\prime}$. If names $\left(\Phi_{\mathrm{sd}}\right) \cap$ $\mathcal{A} \subseteq\left\{v_{0}, p_{0}, e_{1}, e_{2}\right\}$ then the result is immediate. Otherwise, let us consider a bijective renaming $\sigma$ that allows one to rename agent names occurring in $\left(\right.$ names $\left.\left(\Phi_{\text {sd }}^{\prime}\right) \cap \mathcal{A}\right) \backslash\left\{v_{0}, p_{0}, e_{1}, e_{2}\right\}$ to fresh constants in $\Sigma_{0}$. By fresh, we mean that these constants do not occur in $\mathcal{P}_{\text {prox }}$ and $\mathcal{I}_{0}$. We have that $P_{\text {sd }}^{\prime} \sigma$ is a semidishonest prover with frame $\Phi_{\mathrm{sd}}^{\prime} \sigma$.

By construction we have that names $\left(\Phi_{\text {sd }}^{\prime} \sigma\right) \cap \mathcal{A} \subseteq\left\{v_{0}, p_{0}, e_{1}, e_{2}\right\}$ and thus our hypothesis applies. We have that there exists a valid initial configuration $K$ for $\mathcal{P}_{\text {prox }}$ w.r.t. $\mathcal{T}_{\mathrm{MF}}^{0}$ and $\Phi_{\mathcal{I}_{0}}^{\mathcal{T}_{\mathrm{MF}}^{0}} \cup \Phi_{\mathrm{sd}}^{\prime} \sigma$ such that 


$$
\text { exec }=K \stackrel{\text { tr }}{\rightarrow} \mathcal{T}_{\mathrm{MF}}^{0}\left(\left\lfloor\text { end }\left(v_{0}, p_{0}\right)\right\rfloor_{v_{0}}^{t_{v}} \uplus \mathcal{P} ; \Phi ; t\right) .
$$

Applying the renaming $\sigma^{-1}$ along exec, we obtain that:

$$
K \sigma^{-1} \stackrel{\operatorname{tr} \sigma^{-1}}{\longrightarrow} \mathcal{T}_{\text {MF }}^{0}\left(\left\lfloor\text { end }\left(v_{0}, p_{0}\right)\right\rfloor_{v_{0}}^{t_{v}} \uplus \mathcal{P} \sigma^{-1} ; \Phi \sigma^{-1} ; t\right)
$$

Moreover, we know that $K$ is a valid initial configuration for $\mathcal{P}_{\text {prox }}$ w.r.t. $\mathcal{T}_{\mathrm{MF}}^{0}$ and $\Phi_{\mathcal{I}_{0}}^{\mathcal{T}_{\text {MF }}^{0}} \cup \Phi_{\text {sd }}^{\prime} \sigma$ and thus $K=\left(\mathcal{Q} ; \Phi_{\mathcal{I}_{0}}^{\mathcal{T}_{\text {MF }}^{0}} \cup \Phi_{\text {sd }}^{\prime} \sigma ; 0\right)$ where $\mathcal{Q}$ is a multiset of extended processes such that for all $\left\lfloor P^{\prime}\right\rfloor_{a}^{0} \in \mathcal{Q}$, we have that $a \in\left\{v_{0}, p_{0}, e_{1}, e_{2}\right\}$ and there exists $b \in\left\{v_{0}, p_{0}, e_{1}, e_{2}\right\}$ such that $P^{\prime}=\mathrm{P}(a, b)$ or $P^{\prime}=\mathrm{V}(a, b)$. Since $\sigma$ only replace agent names by fresh constants, we can conclude that $\mathcal{Q} \sigma^{-1}=\mathcal{Q}$ and $\Phi_{\mathcal{I}_{0}}^{\mathcal{T}_{\mathrm{MF}}^{0}} \sigma^{-1}=\Phi_{\mathcal{I}_{0}}^{\mathcal{T}_{\mathrm{MF}}^{0}}$. Finally we have:

$$
\left(\mathcal{P} ; \Phi_{\mathcal{I}_{0}}^{\mathcal{T}_{\mathrm{MF}}^{0}} \cup \Phi_{\mathrm{sd}}^{\prime} \sigma \sigma^{-1} ; 0\right) \stackrel{\operatorname{tr} \sigma^{-1}}{\longrightarrow} \mathcal{T}_{\mathrm{MF}}^{0}\left(\left\lfloor\operatorname{end}\left(v_{0}, p_{0}\right)\right\rfloor_{v_{0}}^{t_{v}} \uplus \mathcal{P} \sigma^{-1} ; \Phi \sigma^{-1} ; 0\right) .
$$

with $\left(\mathcal{P} ; \Phi_{\mathcal{I}_{0}}^{\mathcal{T}_{\mathrm{MF}}^{0}} \cup \Phi_{\text {sd }}^{\prime} \sigma \sigma^{-1} ; 0\right)$ a valid initial configuration for $\mathcal{P}_{\text {prox }}$ w.r.t. $\Phi_{\mathcal{I}_{0}}^{\mathcal{T}_{\mathrm{MF}}^{0}} \cup \Phi_{\text {sd }}^{\prime}$. This concludes the proof.

Then, assuming that $\Phi_{\text {sd }}$ does not contain agent names other than $v_{0}, p_{0}, e_{1}$, and $e_{2}$, as a corollary of the result stated and proved in [14], we have the following lemma.

Lemma 2. Let $\mathcal{P}_{\text {prox }}$ be an $\mathcal{I}_{0}$-executable protocol w.r.t. some template $\mathcal{I}_{0}$. Let $P_{\mathrm{sd}}$ be a semi-dishonest prover with frame $\Phi_{\mathrm{sd}}$ such that names $\left(\Phi_{\mathrm{sd}}\right) \cap \mathcal{A} \subseteq$ $\left\{v_{0}, p_{0}, e_{1}, e_{2}\right\}$. Let $\mathcal{T} \in \mathcal{C}_{\mathrm{MF}}$ be a topology and $K$ be a valid initial configuration for $\mathcal{P}_{\text {prox }}$ w.r.t. $\mathcal{T}$ and $\Phi_{\mathcal{I}_{0}}^{\mathcal{T}} \cup \Phi_{\text {sd }}$ such that

$$
K \stackrel{\operatorname{tr}}{\rightarrow} \mathcal{T}\left(\left\lfloor\text { end }\left(v_{0}, p_{0}\right)\right\rfloor_{v_{0}}^{t_{v}} \uplus \mathcal{P} ; \Phi ; t\right) \text { with } \operatorname{Dist}_{\mathcal{T}}\left(v_{0}, p_{0}\right) \geq t_{0} .
$$

We have that there exists $K^{\prime}$ a valid initial configuration $K^{\prime}$ for $\mathcal{P}_{\text {prox }}$ w.r.t. $\mathcal{T}_{\mathrm{MF}}^{0}$ and $\Phi_{\mathcal{I}_{0}}^{\mathcal{T}_{\mathrm{MF}}^{0}} \cup \Phi_{\mathrm{sd}}$ such that

$$
K^{\prime} \stackrel{\operatorname{tr}^{\prime}}{\longrightarrow} \mathcal{T}_{\mathrm{MF}}^{0}\left(\left\lfloor\operatorname{end}\left(v_{0}, p_{0}\right)\right\rfloor_{v_{0}}^{t_{v}^{\prime}} \uplus \mathcal{P}^{\prime} ; \Phi^{\prime} ; t^{\prime}\right) .
$$

Proof. The proof is the same as the one presented in [14] and proceeds as follows:

(1) The exact same trace can be executed in a topology in which all the agents but $v_{0}$ and $p_{0}$ are considered dishonest;

(2) Thanks to the $\mathcal{I}_{0}$-executability of the protocol, all the processes executed by dishonest agents are removed from the initial configuration and the event end $\left(v_{0}, p_{0}\right)$ is still reachable;

(3) Starting with a simpler configuration, the previous trace can still be executed in the simple topology $\mathcal{T}_{\mathrm{MF}}^{0}$;

(4) The last step consists in reducing the initial frame mapping any agent name $a \notin\left\{v_{0}, p_{0}, e_{1}, e_{2}\right\}$ to $e_{1}$. The resulting frame corresponds to $\Phi_{\mathcal{I}_{0}}^{\mathcal{T}_{\mathrm{MF}}^{0}} \cup \Phi_{\mathrm{sd}} \rho$ where $\rho$ replaces agent names other than $v_{0}, p_{0}, e_{2}$ by $e_{1}$. To finish our proof we note that $\Phi_{\text {sd }} \rho=\Phi_{\text {sd }}$ because names $\left(\Phi_{\text {sd }}\right) \cap \mathcal{A} \subseteq\left\{v_{0}, p_{0}, e_{1}, e_{2}\right\}$ by hypothesis. 
Theorem 1. Let $\mathcal{P}_{\text {prox }}$ be an $\mathcal{I}_{0}$-executable protocol w.r.t. some template $\mathcal{I}_{0}$. We have that $\mathcal{P}_{\text {prox }}$ is terrorist fraud resistant w.r.t. $t_{0}$-proximity, if and only if, for all semi-dishonest prover $P_{\text {sd }}$ with frame $\Phi_{\text {sd }}$, there exists a valid initial configuration $K$ for $\mathcal{P}_{\text {prox }}$ w.r.t. $\mathcal{T}_{\mathrm{MF}}^{0}$ and $\Phi_{\mathcal{I}_{0}}^{\mathcal{T}_{\mathrm{MF}}^{0}} \cup \Phi_{\text {sd }}$ such that:

$$
K \stackrel{\operatorname{tr}}{\rightarrow}_{\mathcal{T}_{\mathrm{MF}}^{0}}\left(\left\lfloor\text { end }\left(v_{0}, p_{0}\right)\right\rfloor_{v_{0}}^{t^{\prime}} \uplus \mathcal{P} ; \Phi ; t\right) .
$$

Proof. The $\Leftarrow$ implication is trivial. It is indeed sufficient to choose the topology $\mathcal{T}_{\mathrm{MF}}^{0}$ to conclude. Regarding the $\Rightarrow$ implication, consider first a semi-dishonest prover $P_{\text {sd }}$ with frame $\Phi_{\text {sd }}$ such that names $\left(\Phi_{\text {sd }}\right) \cap \mathcal{A} \subseteq\left\{v_{0}, p_{0}, e_{1}, e_{2}\right\}$, and a valid initial configuration $K$ for $\mathcal{P}_{\text {prox }}$ w.r.t. $\mathcal{T} \in \mathcal{C}_{\mathrm{MF}}$ and $\Phi_{\mathcal{I}_{0}}^{\mathcal{T}} \cup \Phi_{\text {sd }}$ such that:

$$
K \stackrel{\text { tr }}{\rightarrow} \mathcal{T}\left(\left\lfloor\text { end }\left(v_{0}, p_{0}\right)\right\rfloor_{v_{0}}^{t_{v}} \uplus \mathcal{P} ; \Phi ; t\right) \text { with } \operatorname{Dist}_{\mathcal{T}}\left(v_{0}, p_{0}\right) \geq t_{0} .
$$

Applying Lemma 2, we know there exists a valid initial configuration $K^{\prime}$ for P prox w.r.t. $\mathcal{T}_{\mathrm{MF}}^{0}$ and $\Phi_{\mathcal{I}_{0}}^{\mathcal{T}_{\mathrm{MF}}^{0}} \cup \Phi_{\text {sd }}$ such that: $K^{\prime} \stackrel{\operatorname{tr}^{\prime}}{\longrightarrow} \mathcal{T}_{\mathrm{MF}}^{0}\left(\left\lfloor\text { end }\left(v_{0}, p_{0}\right)\right\rfloor_{v_{0}}^{t_{v}^{\prime}} \uplus \mathcal{P}^{\prime} ; \Phi^{\prime} ; t^{\prime}\right)$.

This allows us to conclude. Now, Lemma 1 allows us to conclude that the result holds for any semi-dishonest prover $P_{\text {sd }}$ (even those that rely on agent names outside $\left.\left\{v_{0}, p_{0}, e_{1}, e_{2}\right\}\right)$.

\section{A.2 Proofs of Section 4.2}

Lemma 3. Let $\mathcal{P}_{\text {prox }}$ be a $D B$ protocol. The most general semi-dishonest process $\mathrm{P}^{*}$ together with its associated frame $\Phi^{*}$ is a semi-dishonest prover. Moreover we



$$
\left\{\begin{array}{l}
\left(a_{1}, \operatorname{out}\left(m_{1}\right)\right) \cdot\left(b_{1}, \operatorname{in}\left(m_{1}\right)\right) \ldots\left(a_{i_{0}-1}, \operatorname{out}\left(m_{i_{0}-1}\right)\right) \cdot\left(b_{i_{0}-1}, \operatorname{in}\left(m_{i_{0}-1}\right)\right) . \\
\left(p_{0}, \operatorname{out}\left(m_{i_{0}+1}^{1}\right)\right) \ldots \ldots\left(p_{0}, \operatorname{out}\left(m_{i_{0}}^{k}\right)\right) . \\
\left(v_{0}, \operatorname{out}\left(m_{i_{0}}\right)\right) \cdot\left(v_{0}, \operatorname{in}<2 \times t_{0}\left(m_{i_{0}+1}\right)\right) \\
\left(p_{0}, \operatorname{in}\left(m_{i_{0}}\right)\right) \cdot\left(p_{0}, \operatorname{out}\left(m_{i_{0}+1}\right)\right) . \\
\left(a_{i_{0}+2}, \operatorname{out}\left(m_{i_{0}+2}\right) \cdot\left(b_{i_{0}+2}, \operatorname{in}\left(m_{i_{0}+2}\right)\right) \ldots\left(a_{n}, \operatorname{out}\left(m_{n}\right) \cdot\left(b_{n}, \operatorname{in}\left(m_{n}\right)\right)\right.\right.
\end{array}\right.
$$

where:

$-\left\{a_{i}, b_{i}\right\}=\left\{v_{0}, p_{0}\right\}$ for any $i \in\{1, \ldots, n\} \backslash\left\{i_{0}, i_{0}+1\right\}$;

- $m_{i_{0}+1}=C_{\mathcal{P}}\left[m_{i_{0}}, m_{i_{0}+1}^{1}, \ldots, m_{i_{0}+1}^{p}\right]$

- $\left(x_{1}, \ldots, x_{k}\right) \theta_{\mathcal{P}} \downarrow \sigma=m_{i_{1}}, \ldots, m_{i_{k}}$ where $x_{1}, \ldots, x_{k}$ are the variables occurring in input in the role $\mathrm{V}\left(v_{0}, p_{0}\right)$, and $i_{1}, \ldots, i_{k}$ are the indices among $1, \ldots, n$ corresponding to input performed by $v_{0}$, and $\sigma$ a bijective renaming from variables to names freshly generated by $\mathrm{P}^{*}$ when executing tr$^{*}$.

Proof. This proof strongly relies on Definition 7. First, remark that tr $^{*}$ corresponds to the trace tr in which the extra outputs of $\mathrm{P}^{*}$ are executed just before the $i_{0}{ }^{\text {th }}$ communication action i.e. the output of the challenge; and the answer to the challenge received by $v_{0}$ is anticipated. We show that this sequence of actions $\operatorname{tr}^{*}$ is an execution w.r.t. our semantics:

- $1^{\text {st }}$ line of actions: The actions can be executed following our semantics applying a Tim rule with a delay $\delta=t_{0}$ before each input. Indeed this delay enables the agent $b_{i}$ to receive the message $m_{i}$ sent by $a_{i}$. Moreover, since there is no guarded input the IN rule always applies. 
- $2^{\text {nd }}$ line of actions: It only contains outputs and thus can trivially be executed.

- $3^{\text {rd }}$ line of actions: Before executing the output, we apply a Tim rule to let available all the previous messages (including $\left.m_{i_{0}+1}^{1}, \ldots, m_{i_{0}+1}^{k} s\right)$ for the malicious agent $e$. Since $C_{\mathcal{P}}$ only contains public symbols of functions (otherwise there is a contradiction with item (iv) of Definition 7), we have that $R=C_{\mathcal{P}}\left[\mathrm{w}_{i_{0}}, \mathrm{w}_{i_{0}+1}^{1}, \ldots, \mathrm{w}_{i_{0}+1}^{k}\right]$ where $\mathrm{w}_{i_{0}}$ is the frame variable binding $m_{i_{0}}$ and $\mathrm{w}_{i_{0}+1}^{j},(1 \leq j \leq k)$ is the frame variable binding $m_{i_{0}+1}^{j}$, is a recipe deducing $m_{i_{0}+1}$. Finally the guarded input can be executed because w.l.o.g. we may assume that the reset action has been made right before the output of $m_{i_{0}}$.

$-4^{\text {th }}$ and $5^{\text {th }}$ lines of actions: These actions can be executed for the same reason as the first line applying a TIM rule with a delay $\delta=t_{0}$ before each input.

We denote by $s t(t)$ the set of syntactic subterm of a term $t$.

Lemma 4. Let $t_{0}$ be a term such that $t_{0} \downarrow={ }_{\mathrm{E}} \mathrm{f}\left(u_{1}, \ldots, u_{k}\right)$ with $\mathrm{f}$ a quasi-free function symbol. We have that there exist $u_{1}^{\prime}, \ldots, u_{k}^{\prime}$ such that $\mathrm{f}\left(u_{1}^{\prime}, \ldots, u_{k}^{\prime}\right) \in$ st $\left(t_{0}\right)$ and $u_{i}^{\prime} \downarrow=\mathrm{E} u_{i}$ for any $i \in\{1, \ldots, k\}$.

Proof. Let $t_{0}$ be a term, and $t_{0} \downarrow$ its normal form. Therefore, we have that $t_{0} \rightarrow$ $t_{1} \rightarrow t_{2} \ldots \rightarrow t_{n}=t_{0} \downarrow$. We prove the result by induction on the length $n$ of this derivation.

Base case: $n=0$. We have that $t_{0} \downarrow=t_{0}$, and thus $t_{0}={ }_{\mathrm{E}} \mathrm{f}\left(u_{1}, \ldots, u_{k}\right)$. Since our theory is non-trivial, and since $\mathrm{f}$ does not occur in equations in $\mathrm{E}$, we have that there exists $u_{1}^{\prime}, \ldots, u_{k}^{\prime}$ such that $\mathrm{f}\left(u_{1}^{\prime}, \ldots, u_{k}^{\prime}\right) \in \operatorname{st}\left(t_{0}\right)$ and $u_{i}^{\prime}={ }_{\mathrm{E}} u_{i}$ for any $i \in\{1, \ldots, k\}$. Note that $u_{1}^{\prime}, \ldots, u_{k}^{\prime}$ are in normal form since $t_{0}$ is in normal form, and thus the result holds.

Induction step. In such a case, applying our induction hypothesis, we know that there exist $u_{1}^{\prime}, \ldots, u_{k}^{\prime}$ such that $\mathrm{f}\left(u_{1}^{\prime}, \ldots, u_{k}^{\prime}\right) \in \operatorname{st}\left(t_{1}\right)$ and $u_{i \downarrow}^{\prime} \downarrow={ }_{\mathrm{E}} u_{i}$ for any $i \in\{1, \ldots, k\}$. We denote $\mathrm{g}\left(v_{1}^{\prime}, \ldots, v_{\ell}^{\prime}\right) \rightarrow v^{\prime}$ the rewrite rule applied at position $p$ to rewrite $t_{0}$ in $t_{1}$. We have that there exists a substitution $\theta$ such that $\left.t_{0}\right|_{p}=\mathrm{E}$ $g\left(v_{1}^{\prime}, \ldots, v_{k}^{\prime}\right) \theta$ and $t_{1}=t_{0}\left[v^{\prime} \theta\right]_{p}$. We have that $\mathrm{f}\left(u_{1}^{\prime}, \ldots, u_{n}^{\prime}\right) \in \operatorname{st}\left(t_{1}\right)$, and we distinguish two cases depending on the position $p_{\mathrm{f}}$ at which this subterm occurs in $t_{1}=t_{0}[v \theta]_{p}$ :

1. $p_{\mathrm{f}}$ is a position in $t_{0}[]$. In such a case, we have that either $\mathrm{f}\left(u_{1}^{\prime}, \ldots, u_{n}^{\prime}\right) \in$ $s t\left(t_{0}\right)$ (in case $p_{\mathrm{f}}$ is not a prefix of $p$ ); or $\left.t_{0}\right|_{p_{\mathrm{f}}}=\mathrm{f}\left(u_{1}^{\prime \prime}, \ldots, u_{n}^{\prime \prime}\right)$ for some $u_{1}^{\prime \prime}, \ldots, u_{n}^{\prime \prime}$, and we have that $\left.t_{0}\right|_{p_{\mathrm{f}}} \rightarrow \mathrm{f}\left(u_{1}^{\prime}, \ldots, u_{n}^{\prime}\right)$ with $u_{i}^{\prime \prime}=u_{i}^{\prime}$ or $u_{i}^{\prime \prime} \rightarrow u_{i}^{\prime}$. Therefore, we have that there exist $u_{1}^{\prime \prime}, \ldots, u_{n}^{\prime \prime}$ such that $\mathrm{f}\left(u_{1}^{\prime}, \ldots, u_{n}^{\prime}\right) \in$ $s t\left(t_{0}\right)$ and $u_{i}^{\prime \prime} \downarrow=\mathrm{E} u_{i}$ for any $i \in\{1, \ldots, k\}$.

2. $p_{\mathrm{f}}$ is a position below $p$, i.e. $p$ is a prefix of $p_{\mathrm{f}}$. In such a case, since $\mathrm{f}$ does not occur in $v^{\prime}$ (by definition of quasi-free), we have that $\mathrm{f}\left(u_{1}^{\prime}, \ldots, u_{k}^{\prime}\right) \in \operatorname{st}(x \theta)$ for some $x \in \operatorname{vars}\left(v^{\prime}\right) \subseteq \operatorname{vars}\left(v_{1}^{\prime}, \ldots, v_{\ell}^{\prime}\right)$. Therefore, we have that there exists $t^{\prime}=\left.\mathrm{E} t_{0}\right|_{p}$ such that $\mathrm{f}\left(u_{1}^{\prime}, \ldots, u_{k}^{\prime}\right) \in \operatorname{st}\left(t^{\prime}\right)$. Since we only consider non-trivial 
theory, and since $f$ does not occur in equations in $E$, we have that there exists $u_{1}^{\prime \prime}, \ldots, u_{k}^{\prime \prime}$ such that $\mathrm{f}\left(u_{1}^{\prime \prime}, \ldots, u_{k}^{\prime \prime}\right) \in \operatorname{st}\left(\left.t_{0}\right|_{p}\right)$ and $u_{i}^{\prime \prime}={ }_{\mathrm{E}} u_{i}^{\prime}$ for any $i \in\{1, \ldots, k\}$. Note that $u_{1}^{\prime \prime}, \ldots, u_{k}^{\prime \prime}$ are in normal form since any subterm of $\left.t_{0}\right|_{p}$ is in normal form. Therefore, we have that there exist $u_{1}^{\prime \prime}, \ldots, u_{n}^{\prime \prime}$ such that $\mathrm{f}\left(u_{1}^{\prime \prime}, \ldots, u_{n}^{\prime \prime}\right) \in \operatorname{st}\left(t_{0}\right)$ and $u_{i}^{\prime \prime} \downarrow={ }_{\mathrm{E}} u_{i}$ for any $i \in\{1, \ldots, k\}$.

This concludes the proof.

Lemma 5. Let $\Phi$ be a frame and $c \in \mathcal{N}$ such that $c \notin s t(i m g(\Phi))$, and $\Phi^{+}=$ $\Phi \cup\left\{\mathrm{w}_{c} \stackrel{v_{0}, t_{0}}{\longrightarrow} c\right\}$. Let $R$ be a recipe such that $R \Phi^{+} \downarrow==_{\mathrm{E}} u$. Let $C$ be a context of minimal size made of quasi-free public function symbols such that $u=$ $C\left[c, u_{1}, \ldots, u_{p}\right]$ for some $u_{1}, \ldots, u_{p}$ and $c$ does not occur in $\operatorname{st}\left(\left\{u_{1}, \ldots, u_{p}\right\}\right)$. For any $i \in\{1, \ldots, p\}$, we have that there exists $R_{i}$ such that $R_{i} \Phi^{+} \downarrow={ }_{\mathrm{E}} u_{i}$.

Proof. We prove this result by structural induction on the context $C$.

Base case: $C$ is empty. In such a case, we have that either $p=0$; or $p=1$ with $u=u$. In both case, the result trivially holds.

Inductive case: $C=\mathrm{f}\left(C_{1}, \ldots, C_{k}\right)$. In such a case, by minimality of $C$, we know that $c$ occurs in $u$. We have that $u=\mathrm{f}\left(u_{1}^{\prime}, \ldots, u_{k}^{\prime}\right)$ and for all $i \in\{1, \ldots, k\}$ we note $\left\{u_{1}^{i}, \ldots, u_{p_{i}}^{i}\right\} \subseteq\left\{u_{1}, \ldots, u_{p}\right\}$ the set of terms involved in the sub-context $C_{i}$ i.e. $C_{i}\left[u_{1}^{i}, \ldots, u_{p_{i}}^{i}\right]=u_{i}^{\prime}$. Note that $\bigcup_{1 \leq i \leq k}\left\{u_{1}^{i}, \ldots, u_{p_{i}}^{i}\right\}=\left\{u_{1}, \ldots, u_{p}\right\}$.

Applying Lemma 4 on $R \Phi^{+}$, we deduce that there exist $v_{1}, \ldots, v_{k}$ such that $\mathrm{f}\left(v_{1}, \ldots, v_{k}\right) \in \operatorname{st}\left(R \Phi^{+}\right)$and $v_{i \downarrow}=_{\mathrm{E}} u_{i}^{\prime}$ for any $i \in\{1, \ldots, k\}$. Now, since $c$ occurs in $u$ we have that there exists $i_{0} \in\{1, \ldots, k\}$ such that $u_{1}^{i_{0}}=c$. Therefore we have that $c$ occurs in $v_{i_{0}} \downarrow$ because $C_{i_{0}}$ only contains quasi-free function symbols (i.e. function symbols which do not occur in E). By consequence we have that $\mathrm{f}\left(v_{1}, \ldots, v_{k}\right) \notin i m g\left(\Phi^{+}\right)$and thus there exists $R_{1}, \ldots, R_{k}$ such that $\mathrm{f}\left(R_{1}, \ldots, R_{k}\right) \in \operatorname{st}(R)$ with $R_{i} \Phi^{+} \downarrow={ }_{\mathrm{E}} u_{i}^{\prime}$ for any $1 \leq i \leq k$.

Our induction hypothesis applies for any $i \in\{1, \ldots, k\}$ and we obtain that for all $v \in\left\{u_{1}^{i}, \ldots, u_{p_{i}}^{i}\right\}$, there exists a recipe $R_{v}$ such that $R_{v} \Phi^{+} \downarrow={ }_{E} v$. Considering the previous remark stating that $\bigcup_{1 \leq i \leq k}\left\{u_{1}^{i}, \ldots, u_{p_{i}}^{i}\right\}=\left\{u_{1}, \ldots, u_{p}\right\}$, this allows us conclude the proof.

Proposition 3. Let $\mathcal{P}_{\text {prox }}$ be a DB protocol, and $\mathrm{P}^{*}$ be its most general semidishonest prover with $\Phi^{*}$ its associated frame. Let exec* be the execution witnessing the fact that $\mathrm{P}^{*}$ together with $\Phi^{*}$ is a semi-dishonest prover (as given in Lemma 3). We have that exec* is as follows:

$\operatorname{exec}^{*}:\left(\left\{\left\lfloor\mathrm{V}\left(v_{0}, p_{0}\right)\right\rfloor_{v_{0}}^{0},\left\lfloor\mathrm{P}^{*}\right\rfloor{ }_{p_{0}}^{0}\right\} ; \emptyset ; 0\right) \stackrel{\operatorname{tr}^{*}}{\longrightarrow} \mathcal{T}_{\text {simple }}^{0}\left(\left\{\left\lfloor\operatorname{end}\left(v_{0}, p_{0}\right)\right\rfloor_{v_{0}}^{t_{v}^{*}},\lfloor 0\rfloor_{p_{0}}^{t_{p}^{*}}\right\} ; \Phi^{*} ; t^{*}\right)$

Let $\mathrm{P}_{\mathrm{sd}}$ be a semi-dishonest prover for $\mathcal{P}_{\text {prox }}$ together with its associated frame $\Phi_{\mathrm{sd}}$, and exec be the execution witnessing this fact, i.e.

exec : $\left(\left\{\left\lfloor\mathrm{V}\left(v_{0}, p_{0}\right)\right\rfloor_{v_{0}}^{0},\left\lfloor\mathrm{P}_{\text {sd }}\right\rfloor p_{0}^{0}\right\} ; \emptyset ; 0\right) \stackrel{\operatorname{tr}}{\rightarrow} \mathcal{T}_{\text {simple }}^{0}\left(\left\{\left\lfloor\right.\right.\right.$ end $\left.\left.\left.\left(v_{0}, p_{0}\right)\right\rfloor v_{v_{0}}^{t_{v}},\lfloor 0\rfloor p_{p_{0}}^{t_{p}}\right\} ; \Phi_{\text {sd }} ; t\right)$

We have that there exists a substitution $\sigma: \mathcal{N} \rightarrow \mathcal{T}\left(\Sigma_{c}^{+}, \mathcal{N} \cup \mathcal{A}\right)$ from names freshly generated by $\mathrm{P}^{*}$ to constructor terms such that 
$i$ if $\left(v_{0}, \operatorname{in}(u)\right) \in \operatorname{tr}^{*}\left(\right.$ resp. $\left.\left(v_{0}, \operatorname{in}^{<t}(u)\right) \in \operatorname{tr}^{*}\right)$, then $\left(v_{0}, \operatorname{in}(u \sigma)\right) \in \operatorname{tr}$ (resp. $\left(v_{0}\right.$, in $\left.\left.^{<t}(u \sigma)\right) \in \operatorname{tr}\right)$.

ii if $(a$, out $(u)) \in \operatorname{tr}^{*}$ for some $a \in \mathcal{A}$, then $R \Phi_{\mathrm{sd}} \downarrow={ }_{\mathrm{E}} u \sigma$ for some recipe $R$.

Proof. Along an execution, variables occurring in input as well as those occurring in a let instruction are instantiated. We denote by $\tau_{\mathrm{tr}}$ the substitution associated to a given execution tr. We now establish each item separately.

Item (i): We consider $\mathrm{V}\left(v_{0}, p_{0}\right)=\mathrm{a}_{1} \ldots \mathrm{a}_{k}$. Because $\left(v_{0}\right.$, in $\left.{ }^{\star}(u)\right) \in \operatorname{tr}^{*}$, we have that there exists $i_{0} \in\{1, \ldots, k\}$ such that $a_{i_{0}}=\operatorname{in}^{\star}(x)$ for some $x \in \mathcal{X}$ and $x \tau_{\text {tr* }}=u$. By Lemma 3, we have that there exists $\tau: \mathcal{X} \mapsto \mathcal{N}$ a bijective renaming from variables to names freshly generated by $\mathrm{P}^{*}$ such that $u=x \theta_{\mathcal{P}} \downarrow \tau$.

On the other side, we know that the process has been entirely executed in $\mathrm{tr}$ and therefore there exists $\left(v_{0}\right.$, in $\left.^{\star}\left(u^{\prime}\right)\right) \in \operatorname{tr}$ such that $u^{\prime}=x \tau_{\text {tr }}$ and thus by definition of $\theta_{\mathcal{P}}$, there exists $\sigma$ such that $u^{\prime}=_{\mathrm{E}} x \theta_{\mathcal{P}} \downarrow \sigma$. By consequence we have that $u^{\prime}=x \tau_{\mathrm{tr}}={ }_{\mathrm{E}} x \theta_{\mathcal{P} \downarrow} \sigma=\left(u \tau^{-1}\right) \sigma$. We conclude choosing $\sigma^{\prime}=\tau^{-1} \sigma$.

Item (ii): We have that $(a$, out $(u)) \in \operatorname{tr}^{*}$. We distinguish several cases depending on the origin of this output.

In case $a=v_{0}$, it is an immediate corollary of item (i). Indeed we can prove by induction on the length of $\operatorname{tr}$ that for each configuration $K$ in exec, there exists a configuration $K^{*}$ in exec* such that if we note $V^{*}$ the process executed by $v_{0}$ in $K^{*}$ then $V^{*} \sigma$ is the process executed by $v_{0}$ in $K$.

Now, we assume that $a=p_{0}$, and we distinguish two cases depending on whether $u=m_{i_{0}+1}^{j}(1 \leq j \leq k)$ or not. If not, applying Lemma 3 , we have that $\left(v_{0}, \operatorname{in}(u)\right) \in \operatorname{tr}^{*}\left(\right.$ or $\left(v_{0}\right.$, in $\left.\left.^{<t}(u)\right) \in \operatorname{tr}^{*}\right)$, and applying item (i) we have know that $\left(v_{0}\right.$, in $\left.(u \sigma)\right) \in \operatorname{tr}\left(\right.$ or $\left(v_{0}\right.$, in $\left.\left.^{<t}(u \sigma)\right) \in \operatorname{tr}\right)$. Therefore, we know that there exists a recipe $R$ such that $R \Phi_{\text {sd } \downarrow}={ }_{E} u \sigma$.

Now, we assume that $u=m_{i_{0}+1}^{j}$ for some $j \in\{1, \ldots, k\}$. Thanks to Lemma 3, we know that $\left(v_{0}\right.$, in $\left.^{<2 t_{0}}\left(m_{i_{0}+1}\right)\right) \in \operatorname{tr}^{*}$ and $m_{i_{0}+1}=C_{\mathcal{P}}\left[m_{i_{0}}, m_{i_{0}+1}^{1}, \ldots, m_{i_{0}+1}^{k}\right]=$ $x \theta_{\mathcal{P} \downarrow} \tau$ for some bijective renaming $\tau: \mathcal{X} \mapsto \mathcal{N}$ from variables to names freshly generated by $\mathrm{P}^{*}$.

Applying item (i), we have that $\left(v_{0}\right.$, in $\left.{ }^{<2 t_{0}}\left(m_{i_{0}+1} \sigma\right)\right) \in \operatorname{tr}$. Moreover, following the hypotheses on the structure of $\mathrm{V}\left(v_{0}, p_{0}\right)$, we know that in $\operatorname{tr}$ there is a unique output executed by $v_{0}$ before this guarded input that contains the challenge. In addition, $p_{0}$ cannot receive the challenge soon enough to make an output containing $c$ available to fill the guarded input. Indeed, assume that it was the case, and let $t_{\text {reset }}$ (resp. $t_{\text {out }}$ and $t_{\text {in }}$ ) the time when the reset action (resp. output of the challenge, reception of the guarded input) is executed in $\mathrm{tr}$ then we have that:

$$
t_{\text {in }} \geq t_{\text {out }}+2 \times \operatorname{Dist}_{\mathcal{T}_{\text {oracle }}}\left(v_{0}, p_{0}\right) \geq t_{\text {reset }}+2 \times \operatorname{Dist}_{\mathcal{T}_{\text {oracle }}}\left(v_{0}, p_{0}\right)=t_{\text {reset }}+2 \times t_{0} .
$$

This is in contradiction with the constraint imposed by the guarded input: $t_{\text {reset }}-t_{\text {in }}<2 \times t_{0}$. Finally, we deduce that there exists $\Phi^{+}=\Phi \cup\left\{\mathrm{w} \stackrel{v_{0}, t_{\text {out }}}{\longrightarrow} c\right\}$ such that $c \notin s t(i m g(\Phi))$ and a recipe $R$ such that $R \Phi^{+} \downarrow={ }_{\mathrm{E}} m_{i_{0}+1} \sigma$. Lemma 5 applies and we conclude that there exists a recipe $R_{u}$ such that $R_{u} \Phi^{+} \downarrow={ }_{\mathrm{E}}$ $m_{i_{0}+1}^{j} \sigma$ and thus $R_{u} \Phi_{\text {sd } \downarrow}={ }_{\mathrm{E}} u \sigma$ since $\Phi^{+}$is a subframe of $\Phi_{\text {sd }}$. 
Theorem 2. Let $\mathcal{P}_{\text {prox }}$ be a $D B$ protocol and $\mathcal{I}_{0}$ be a template. Let $\Phi^{*}$ be the frame associated to the most general semi-dishonest prover of $\mathcal{P}_{\text {prox }}$. We have that $\mathcal{P}_{\text {prox }}$ is terrorist fraud resistant w.r.t. $t_{0}$-proximity if, and only if, there exists a topology $\mathcal{T}=\left(\mathcal{A}_{0}, \mathcal{M}_{0}\right.$, Loc $\left._{0}, v_{0}, p_{0}\right) \in \mathcal{C}_{\mathrm{MF}}$ and a valid initial configuration $K_{0}$ for $\mathcal{P}_{\text {prox }}$ w.r.t. $\mathcal{T}$ and $\Phi^{*} \cup \Phi_{\mathcal{I}_{0}}^{\mathcal{T}}$ such that:

$$
K_{0} \stackrel{\operatorname{tr}}{\rightarrow} \mathcal{T}\left(\left\lfloor\text { end }\left(v_{0}, p_{0}\right)\right\rfloor v_{0}^{t_{0}^{\prime}} \uplus \mathcal{P} ; \Phi ; t\right) \text { with } \operatorname{Dist}_{\mathcal{T}}\left(v_{0}, p_{0}\right) \geq t_{0} .
$$

Proof. The direct implication is trivial, and we therefore concentrate on the other one. Let $\mathrm{P}_{\mathrm{sd}}$ be a semi-dishonest prover for $\mathcal{P}_{\text {prox }}$ with its associated frame $\Phi_{\text {sd }}$, and $\operatorname{tr}$ be the trace witnessing this fact. We denote $\mathrm{P}^{*}$ the most general semidishonest prover, $\Phi^{*}$ its associated frame, and $\operatorname{tr}^{*}$ the trace witnessing this fact.

Applying Proposition 3, there exists a substitution $\sigma: \mathcal{N} \rightarrow \mathcal{T}\left(\Sigma_{c}^{+}, \mathcal{N} \cup \mathcal{A}\right)$, from names freshly generated by $\mathrm{P}^{*}$ in $\operatorname{tr}^{*}$ to constructor terms such that:

$\mathrm{i}$ if $\left(v_{0}, \operatorname{in}(u)\right) \in \operatorname{tr}^{*}$, then $\left(v_{0}, \operatorname{in}(u \sigma)\right) \in \operatorname{tr}$.

ii if $(a$, out $(u)) \in \operatorname{tr}^{*}$ for some $a \in \mathcal{A}$, then $R \Phi_{\text {sd } \downarrow}=_{\mathrm{E}} u \sigma$ for some recipe $R$.

By hypothesis, there exist a topology $\mathcal{T} \in \mathcal{C}_{\mathrm{MF}}$ and a valid initial configuration $K^{*}$ for $\mathcal{P}_{\text {prox }}$ w.r.t. $\mathcal{T}$ and $\Phi^{*} \cup \Phi_{\mathcal{I}_{0}}^{\mathcal{T}}$ such that

$$
\left(\mathcal{P}_{\text {init }} ; \Phi_{\mathcal{I}_{0}}^{\mathcal{T}} \cup \Phi^{*} ; 0\right) \stackrel{\operatorname{tr}_{0}}{\longrightarrow} \mathcal{T}\left(\left\lfloor\text { end }\left(v_{0}, p_{0}\right)\right\rfloor_{v_{0}}^{t^{\prime}} \uplus \mathcal{P} ; \Phi_{\mathcal{I}_{0}}^{\mathcal{T}} \cup \Phi^{*} \cup \Phi_{\text {out }} ; t\right)
$$

for some frame $\Phi_{\text {out }}$. Without loss of generality, we may assume that $\mathcal{T}=$ $\left(\mathcal{A}_{0}, \mathcal{M}_{0}, \operatorname{Loc}_{0}, v_{0}, p_{0}\right)$ with $\mathcal{M}_{0} \neq \emptyset$. Otherwise, we add such a malicious agent, and the trace remains executable. Applying the substitution $\sigma$ along this execution, we obtain a valid execution (remember that our calculus does not feature else branches):

$$
\left(\mathcal{P}_{\text {init }} \sigma ; \Phi_{\mathcal{I}_{0}}^{\mathcal{T}} \sigma \cup \Phi^{*} \sigma ; 0\right) \stackrel{\operatorname{tr}_{0} \sigma}{\longrightarrow} \mathcal{T}\left(\left\lfloor\text { end }\left(v_{0}, p_{0}\right)\right\rfloor_{v_{0}}^{t^{\prime}} \uplus \mathcal{P} \sigma ; \Phi_{\mathcal{I}_{0}}^{\mathcal{T}} \sigma \cup \Phi^{*} \sigma \cup \Phi_{\text {out }} \sigma ; t\right) .
$$

Actually, since names occurring in $\operatorname{dom}(\sigma)$ are names freshly generated by $\mathrm{P}^{*}$, we have that $\mathcal{P}_{\text {init }} \sigma=\mathcal{P}_{\text {init }}, \Phi_{\mathcal{I}_{0}}^{\mathcal{T}} \sigma=\Phi_{\mathcal{I}_{0}}^{\mathcal{T}}$, and therefore, we have that:

$$
\left(\mathcal{P}_{\text {init }} ; \Phi_{\mathcal{I}_{0}}^{\mathcal{T}} \cup \Phi^{*} \sigma ; 0\right) \stackrel{\operatorname{tr}_{0} \sigma}{\longrightarrow} \mathcal{T}\left(\left\lfloor\text { end }\left(v_{0}, p_{0}\right)\right\rfloor{ }_{v_{0}}^{t^{\prime}} \uplus \mathcal{P} \sigma ; \Phi_{\mathcal{I}_{0}}^{\mathcal{T}} \cup \Phi^{*} \sigma \cup \Phi_{\text {out }} \sigma ; t\right) .
$$

Finally, from item 2 , we have that for any $u \in i m g\left(\Phi^{*}\right)$, there exists a recipe $R$ such that $R \Phi_{\mathrm{sd}} \downarrow={ }_{\mathrm{E}} u \sigma$. We can thus deduce that for any term $v$ and recipe $R_{v}$ such that $R_{v}\left(\Phi_{\mathcal{I}_{0}}^{\mathcal{T}} \cup \Phi^{*} \sigma\right) \downarrow={ }_{\mathrm{E}} v$ we have that there exists $R_{v}^{\prime}$ such that $R_{v}^{\prime}\left(\Phi_{\mathcal{I}_{0}}^{\mathcal{T}} \cup \Phi_{\text {sd }}\right) \downarrow==_{\mathrm{E}} v$. Starting by applying a TIM rule with a delay $\delta$ equal to twice the greatest distance between two agents in $\mathcal{T}$, we have:

$$
\left(\mathcal{P}_{\text {init }} ; \Phi_{\mathcal{I}_{0}}^{\mathcal{T}} \cup \Phi_{\text {sd }} ; 0\right) \stackrel{\operatorname{tr}_{0} \sigma}{\longrightarrow} \mathcal{T}\left(\left\lfloor\text { end }\left(v_{0}, p_{0}\right)\right\rfloor_{v_{0}}^{t^{\prime}} \uplus \mathcal{P} \sigma ; \Phi_{\mathcal{I}_{0}}^{\mathcal{T}} \cup \Phi_{\text {sd }} \cup \Phi_{\text {out }}^{\prime} \sigma ; t+\delta\right) .
$$

with $\phi_{\text {out }}^{\prime}=\left\{\mathrm{w} \stackrel{a, t+\delta}{\longrightarrow} u \sigma \mid \mathrm{w} \stackrel{a, t}{\longrightarrow} u \in \Phi_{\text {out }}\right\}$. The delay $\delta$ enables a dishonest agent (there is one by assumption) to build any term occurring in $\Phi^{*} \sigma$ from $\Phi_{\text {sd }}$. 
Corollary 1. Let $\mathcal{P}_{\text {prox }}$ be a DB protocol and $\mathcal{I}_{0}$ be a template such that $\mathcal{P}$ is $\mathcal{I}_{0}$-executable. Let $\mathrm{P}^{*}$ be the most general semi-dishonest prover for $\mathcal{P}$ together with its associated frame $\Phi^{*}$. We have that $\mathcal{P}_{\text {prox }}$ is terrorist fraud resistant w.r.t. $t_{0}$-proximity if, and only if, there exists a valid initial configuration $K_{0}$ for $\mathcal{P}_{\text {prox }}$ w.r.t. $\mathcal{T}_{\mathrm{MF}}^{0}$ and $\Phi^{*} \cup \Phi_{\mathcal{I}_{0}}^{\mathcal{T}_{\mathrm{MF}}^{0}}$ such that:

$$
K_{0} \stackrel{\text { tr }}{\rightarrow} \mathcal{T}_{\mathrm{MF}}^{t_{0}}\left(\left\lfloor\text { end }\left(v_{0}, p_{0}\right)\right\rfloor{ }_{v_{0}}^{t^{\prime}} \uplus \mathcal{P} ; \Phi ; t\right) .
$$

Proof. We distinguish the two directions of the implication.

If $\mathcal{P}_{\text {prox }}$ is terrorist fraud resistant w.r.t. $t_{0}$-proximity then there exist a topology $\mathcal{T} \in \mathcal{C}_{\mathrm{MF}}$ and a valid initial configuration $K$ for $\mathcal{P}_{\text {prox }}$ w.r.t. $\mathcal{T}$ and $\Phi^{*} \cup \Phi_{\mathcal{I}_{0}}^{\mathcal{T}}$ such that:

$$
K \stackrel{\operatorname{tr}}{\rightarrow} \mathcal{T}\left(\left\lfloor\text { end }\left(v_{0}, p_{0}\right)\right\rfloor v_{v_{0}}^{t_{v}} \uplus \mathcal{P} ; \Phi ; t\right) \text { with } \operatorname{Dist}_{\mathcal{T}}\left(v_{0}, p_{0}\right) \geq t_{0} .
$$

Since names $\left(\Phi^{*}\right) \cap \mathcal{A} \subseteq\left\{v_{0}, p_{0}, e_{1}, e_{2}\right\}$, Lemma 2 applies and we obtain that there exits a valid initial configuration $K_{0}$ for $\mathcal{P}_{\text {prox }}$ w.r.t. $\mathcal{T}_{\mathrm{MF}}^{t_{0}}$ and $\Phi^{*} \cup \Phi_{\mathcal{I}_{0}}^{\mathcal{T}}$ such that:

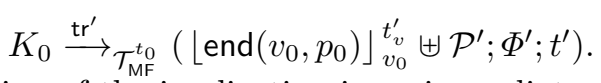

The other direction of the implication is an immediate application of Theorem 2 because $\mathcal{T}_{\mathrm{MF}}^{t_{0}} \in \mathcal{C}_{\mathrm{MF}}$. 